INTERSECTIONALITY IN PROGRAMS AND SERVICES FOR NEWCOMER YOUTH IN TORONTO

\author{
by \\ Fatima Saher, BA, Ryerson University, Toronto, 2016 \\ A Major Research Paper \\ presented to Ryerson University \\ in partial fulfillment of the \\ requirements for the degree of \\ Master of Arts \\ in the Program of \\ Immigration and Settlement Studies
}

Toronto, Ontario, Canada, 2017

C F Fatima Saher 2017 


\section{$\underline{\text { Author's Declaration for Electronic Submission of a Major Research Paper }}$}

I hereby declare that I am the sole author of this MRP. This is a true copy of the MRP, including any required final revisions.

I authorize Ryerson University to lend this MRP to other institutions or individuals for the purpose of scholarly research.

I further authorize Ryerson University to reproduce this MRP by photocopying or by other means, in total or in part, at the request of other institutions or individuals for the purpose of scholarly research.

I understand that my MRP may be made electronically available to the public. 


\title{
INTERSECTIONALITY IN PROGRAMS AND SERVICES FOR NEWCOMER YOUTH IN
} TORONTO

\author{
Fatima Saher \\ Master of Arts, 2017 \\ Immigration and Settlement Studies \\ Ryerson University
}

\begin{abstract}
$\underline{\text { Abstract }}$
Newcomer youth arrive to Canada with multiple social identities including, their immigration pathway, status, race, gender, sexuality etc. which shapes their individual experiences and settlement. The term "intersectionality" was coined by Crenshaw (1991) to highlight how identities intersect and can act as sites of cross-roads of oppression, as rooted in an understanding of social exclusion and inclusion. This paper studies how newcomer youth programs in Toronto are informed by intersectionality. Audio interviews were conducted with three service providers to study their cognizance of intersectionality, their experiences with newcomer youth who have multiple identities, and if/how their programs incorporate an intersectional lens. Results showed that while key informants practice and understand intersectionality, they feel limited by funding and other barriers which stifle their inclusivity. The potential benefits of this project exist for service providers and their workplaces, who can evaluate their understanding of newcomer youth to recognize and support them better.
\end{abstract}

Key words: intersectionality; newcomer youth; Toronto 


\section{Acknowledgments}

I would like to thank my supervisor, Dr. Kiaras Gharabaghi, for his assistance, insight, and kindness in helping me write and complete this MRP. Without his helpful suggestions that expanded my understanding of newcomer youth, I would not find myself in the position that I do. I am similarly thankful for my second reader, Dr. Vappu Tyyskä, for her willingness to be a part of this project and for taking the time out to provide me with such detailed feedback and input. I am grateful that I was able to enrich my understanding on a topic I feel passionately about by working with both professors on this study.

I would also like to thank the participants in this MRP for their meaningful responses and readiness to collaborate. I hope that our conversations and this research proves worthwhile to all newcomer youth and newcomer organizations in Toronto.

I'd like to thank my family and friends for their on-going support which, both spoken and unspoken, sustained my efforts. I cannot imagine having to finish this paper without their ability to encourage, entertain, and put things in perspective when things seemed unconquerable.

To my parents, who provided me with all the tools and resources I needed to succeed as a graduate student-thank you. Your unconditional love, support, and belief in my capability and intellect is more than I could ever ask for.

More so than anything else, I'd like to thank God for giving me the guidance and opportunity to write this MRP. 


\section{Table of Contents}

Abstract $\quad$ iii

Acknowledgments iv

Introduction 1

$\begin{array}{ll}\text { Literature Review } & 3\end{array}$

Theoretical Approach $\quad 3$

Intersectionality $\quad 3$

Social exclusion and inclusion $\quad 5$

$\begin{array}{ll}\text { Intersectionality in organizations } & 7\end{array}$

Homogenizing newcomers when providing services $\quad 7$

Cognizance of multiple identities and issues $\quad 9$

Need for more comprehensive and cooperative service provisions $\quad 11$

$\begin{array}{ll}\text { The role of service providers and workers } & 12\end{array}$

Service providers as resources for insights and critiques $\quad 13$

Barriers when supporting and providing services for newcomer youth 15

Homelessness $\quad 15$

$\begin{array}{ll}\text { Mental health support } & 17\end{array}$

$\begin{array}{ll}\text { Research Questions } & 19\end{array}$

Methodology $\quad 20$

Research design $\quad 20$

Sampling design $\quad 20$

Data collection and analysis

Findings $\quad 24$

Intersectionality $\quad 25$

Participants' definition of intersectionality 25

Personal approaches to service provision $\quad 26$

$\begin{array}{ll}\text { Settlement experiences of newcomer youth } & 27\end{array}$

Barriers when accessing services $\quad 29$

Youth space $\quad 29$

Identity-based limitations for newcomer youth 33

Personal anecdotes of multiple identities $\quad 35$

Organizational approaches to intersectionality 40

$\begin{array}{ll}\text { Initiatives } & 40\end{array}$

$\begin{array}{lr}\text { Funding } & 42\end{array}$

Best practices $\quad 46$

$\begin{array}{ll}\text { Collaborative efforts } & 48\end{array}$

$\begin{array}{ll}\text { Discussion } & \mathbf{5 0}\end{array}$

Service providers 51

Defining intersectionality $\quad 52$

Need for and applying intersectionality $\quad 52$

Newcomer youth organizations and programs 56 
Initiatives within organizations $\quad 56$

Initiatives within the community $\quad 59$

$\begin{array}{ll}\text { Recommendations } & 63\end{array}$

$\begin{array}{ll}\text { Conclusion } & 67\end{array}$

Appendix A: Ryerson Ethics Board Approval Letter 69

$\begin{array}{ll}\text { Appendix B: Recruitment Poster } & 70\end{array}$

Appendix C: Recruitment Script (email and phone)

Appendix D: Interview Guide $\quad 72$

$\begin{array}{ll}\text { References } & 74\end{array}$ 


\section{Introduction}

Newcomer youth represent a significant portion of the overall immigrant/ youth population in Canada. Statistics show that foreign-born youth and Canadian-born youth from immigrant families account for approximately " $20 \%$ of all young Canadians under the age of 18 " and this was expected to reach $25 \%$ by 2016 (Ngo et al., 2015, p. 63). Notwithstanding the limited knowledge of the dominant language/culture and individual pre-settlement experiences (Caxaj \& Berman, 2010, p. 18), newcomer youth are a diverse group who have multifaceted identities and it is imperative that their needs are reflected in service provision. Researchers have studied the settlement experience of newcomer youth in depth and identify that membership in particular identity groups shape the type of service and needs they have. Newcomer youth who cannot identify with the dominant identities of race, class, religion, sexuality, socio-economic status etc. are often marginalized and forced to encounter complex inequalities and systems of oppression. Youth who are members of numerous minority groups are not only marginalized from one affiliation, but can feel discounted by more than one identity simultaneously. Community service organizations that offer services and programs to newcomer youth can be at the forefront of anti-oppression work and challenge inequality for newcomer youth in vital ways. To be successful, community organizations must reflect the intersectional needs of all newcomer youth and be aware of how their multiple identities work together.

Intersectionality is a theory and framework which acknowledges that individual's multiple identities can result in overlapping and intersecting oppressions, discriminations, and marginalization (Crenshaw, 1991). An intersectional perspective examines how individual's experiences of marginalization are the result of a combination of social categories, which are interrelated and shape their lives (Núñez, 2014, p. 86). Because of their flexible identities, 
coupled with struggles or barriers that might arise from their immigration status (Caxaj \& Berman, 2010, p. 28), it is important to apply an intersectional lens when providing services to newcomer youth. Using these ideas, this paper aims to examine the cognizance and comprehension of intersectionality that newcomer youth programs have. It is important for organizations to recognize intersectionality and its potential application in their programs, as well as to take initiatives and steps to be more inclusive of newcomer youth with intersecting identities.

For the purpose of this study, newcomer youth are those who are eligible and often seek services from community organization because they are immigrants, refugee claimants, or have refugee status, are between the ages of 13-24, and have been in Canada for 5 years or less. The goal of this study is to infer, by speaking to three key informants, how intersectionality is understood, valued, and incorporated in newcomer youth programs in Toronto. Key informants, who are service providers in newcomer youth organizations in Toronto, can highlight gaps and discrepancies in service provisions and can provide meaningful responses. They are experts in their fields and can share the practical application of intersectionality in their programs. In-depth audio interviews were conducted with three service providers to study their cognizance of intersectionality, how it informs the support they provide, their experience with newcomer youth who have multiple identities, and if/how their programs incorporate and promote an intersectionality driven approach. 


\section{$\underline{\text { Literature Review }}$}

This section of the paper is a literature review on academic and grey literature which extrapolates how intersecting identities of newcomer youth impact and influence their access to newcomer programs. The aim of this literature review is to gather and analyze the various existing interpretations and applications of intersectionality as it pertains to service provisions for newcomers, and more specifically newcomer youth. First, theories of intersectionality and social exclusion and inclusion are outlined to help contextualize the research questions and aims of this study. Next, the following themes and ideas are explored: intersectionality in organizations, the role of service providers and workers, and lastly, barriers and limitations that arise when

providing services and support to newcomer youth, specifically around homelessness and mental health support.

$\underline{\text { Theoretical approach }}$

\section{Intersectionality}

This paper is embedded in the following theoretical approaches: intersectionality, social exclusion and social inclusion theory. In this study, intersectionality is used to discuss the relationship between multiple identities and inequality, and its application will be more towards the experience of newcomer youth. A core aspect of intersectionality is related to Lee \& Brotman's (2013) definition of social location, which they argue is a combination of an individual's socio-historical /geographic context, and group affiliations that are self-identified and/or socially imposed, such as, race, ethnicity, gender, ability, class, citizenship status, sexual/gender identity (Lee \& Brotman, 2013, p. 171). Intersectionality is defined and applied distinctly by theorists, researchers, service providers, and policymakers alike, but is recognized as a theoretical framework useful to understand the interrelated experiences of people with 
multiple social positions and identities. The term "intersectionality" was first coined by Crenshaw (1991) to highlight the ways in which our identities intersect and can act as sites of cross-roads of oppression. Crenshaw (1991) discusses how an individual's connection or affiliation with multiple parts of their identity are not just interconnected and linked, but often reflect their access to power and privilege. Similarly, Lee \& Brotman (2013), emphasize that an individual's intersecting group affiliations determine their social power, privilege, and penalty on structural and institutional levels (p. 171).

The idea that an individual's race, gender, sexuality, religion, socio-economic status etc. could result in multiple linked experiences with oppression is also alluded to by Suárez-Orozco et al. (2015). They suggest that an intersectional perspective allows the recognition of such disadvantages, which are layered and come with membership in various social categories and identities (Suárez-Orozco et al., 2015, p. 3). Intersectionality suggests against separating distinct identities from one another or merely seeing them as layered or multiple, but rather as complex intersections that function on the same level and field. Likewise, Patricia Hills Collins uses intersectionality to talk about navigating simultaneously marginalized and privileged identities, claiming that both types of identities are crucial to understand interlocking oppression (Núñez, 2016, p. 34). With regards to newcomer youth, Caxaj \& Berman (2010) claim that intersectionality can help contextualize the multiple factors and social positions, such as limited knowledge of the dominant language/culture, war trauma, individual pre-settlement experiences etc., that contribute to feelings of belonging (p. 18). For example, newcomer youth from a lower socio economic status who also identify as members of the LGBTQ community may lack feelings of belonging because they are discriminated against in different spaces and in different 
ways; thus, their lived experience with inequality is shaped as an amalgamation of all their identities.

\section{Social exclusion and inclusion}

To examine how and whether newcomer youth programs work towards providing services that don't reproduce those same barriers, or how they actively work towards helping eliminate such barriers, it is important to comprehend theories of social exclusion and inclusion. Closely tied to ideas of marginalization, discrimination, and barriers that intersectionality proposes, social exclusion and inclusion theory similarly highlight how lack of access to services can affect certain identity groups disproportionately. While social exclusion can be defined in various ways, it is generally referred to as the inability of individuals or groups of people to participate and effectively take part in economic, social, and cultural life due to structural inequalities (Thomas, 2012; Mihai \& Burciu, 2015). Adascalitei (2016) introduces her analysis of social exclusion theory by stating that it is the cumulative effects and experiences of realities such as housing, employment, education, and health care discrimination (p. 10). However, while discussions of those inequalities are often focused on individuals/household, social exclusion is embedded and concerned with the relationships between individuals and society (Mihai \& Burciu, 2015, p. 136).

To situate the discussion of identity, inequality and belonging further, the framework that social exclusion provides is relevant to this paper on newcomer youth. Mihai and Burciu (2015) explain that social exclusion in youth is complex and multidimensional, and is often rooted in social and cultural causes (p. 136). They suggest that youth can experience social exclusion from educational/training barriers and government policies, as well as factors that tend to favour 
specific social categories and identities of race, sex, gender etc. (p. 136). Structural and cultural barriers can influence young people to feel forms of exclusion, or they might even choose to disengage and self-exclude, due to fear of marginalization and discrimination based on their racial, sexual, cultural and other identities (Mihai \& Burciu, 2015, p. 136). Oxman-Martinez \& Choi (2014) argue that the establishment of youth identity is a development that predicts how they view themselves and their future prospects (p. 24). They infer that studying the presence and effects of social exclusion faced by newcomer youth can provide insight into factors that promote a healthy social and academic life (p. 24).

Often discussed in comparison to social exclusion, social inclusion is defined as effort and resistance which aims to break down the exclusive barriers which prevent individuals from participating in society (Thomas, 2012, p. 6-7). Omidvar and Richmond (2003) refer to social inclusion, regarding newcomers, as initiatives which address basic notions of social belonging, acceptance, and recognition. Furthermore, a key aspect and function of social inclusion is the removal of structural and institutional barriers for marginalized communities, such as newcomer immigrants and refugees, in social, economic, and political spheres (Moss, 2008, p. 19). In European context, Moss (2008) cites the emergence of social inclusion as a response to the social exclusion that arose in the form of division in the labour market, inadequate resources, and lack of social services for those who were excluded but in need during the 1980's in Europe. In present context, the usefulness and significance of social inclusion is that it allows for the identification of barriers which hinder newcomer youth integration, settlement process, and access to "specific services, programs and infrastructural changes... [needed] in order to promote social inclusion" (Moss, 2008, p. 19). 
Oxman-Martinez \& Choi (2014) further the discussion of social exclusion and inclusion as it pertains to youth in a different direction. They claim that while measuring the existence and conditions of social inclusion or exclusion has largely been contingent on familial structures, circumstances, income, employment etc., it is just as imperative to apply a critical lens and look beyond adult-focused or family poverty-focused models (p. 24). A critique of both social exclusion and inclusion is that actual lived experiences of exclusion and inclusion are more nuanced and requires analysis that considers multiple disadvantages on different levels. Simply creating a dichotomy of either being included or excluded oversimplifies the complexity and makes it seems as if individuals deal with the same type of disadvantages (Thomas, 2012, p. 9). However, realistically intersecting identities and disadvantages that work in different social categories, such as race, gender, and socio-economic status, can allow groups and individuals to be both included and excluded within society and the categories do not have to be mutually exclusive (Thomas, 2012, p. 9).

\section{$\underline{\text { Intersectionality in organizations }}$}

Research about the functions and organization of newcomer service providers indicates the importance of an intersectional approach. The following section outlines the significance of intersectional identities and discusses what literature suggests are best methods for community organizations and agencies to practice when promoting and sustaining inclusivity.

\section{Homogenizing newcomers when providing services}

An intersectional perspective attempts to emphasize and counter disadvantages, inequalities, and marginalization that might occur from identification of multiple social categories (Núñez, 2014, p. 86). Researchers stress the importance of newcomer organizations to 
actively consider the amalgamation of identities in the clientele they serve and represent, and being cautionary of viewing any identity group as homogenous. Several publications point out the significance of accounting for peoples' holistic identities while emphasizing how misconstrued it is to assume that individuals are synonymous and identical to others in their identity group (Warner \& Shields, 2013; Malcom \& Mendoza, 2014; Kainer, 2016). Instead of highlighting the complexity of individual identities, newcomer service providers might unknowingly and mistakenly distill their understanding or perspective of the clientele, especially if their services are targeted to a specific community, which results in inadequate support lacking a nuanced and intersectional lens.

The downfalls of homogenizing a group are also outlined in Feminism without Borders: Decolonizing Theory, Practicing Solidarity, where Chandra Mohanty (2003) advises against Western discourses that paint and categorize women in postcolonial countries as a homogenous powerless group, and on discourses about women that do not acknowledge the unique experiences of race and ethnicity. Similar sentiments are discussed in a report by Southall Black Sisters (2010) from the UK on how community cohesion policies affect the lives of marginalised ethnic, minority, and newcomer women. This report criticizes the 2001 UK government implementation and shift away from multiculturalism and towards the cohesion and faith agenda; an initiative which promotes and emphasizes assimilation through adopting British values and by practicing religion in the public sphere (Patel \& Siddiqui, 2007, p. 268). It was intended to promote diversity and minimize barriers between community group members, yet serves as a way to use religious based identities to monitor and manage the threat of religious fundamentalism (Patel \& Siddiqui, 2007, p. 268). The Southall Black Sisters (2010) condemn the cohesion and faith agenda by claiming that it falsely promotes a myth of harmonious and 
singular communities, as it does not mention any, "structural inequalities within communities based on differences in class, caste, gender and even different interpretations of the same faith or religion" (Southall Black Sisters, 2010, p. 33). The shortcomings of homogenizing an identity group is that it can lead to generalizations and acceptance of negative stereotypes, which is harmful to both an organization's mandate of inclusivity and the population they serve. Often, dominant discourses do not just paint a homogenous picture which might be skewed from reality, but can also create overarching stereotypes which are regressive and discriminatory. For example, Bauder (2003), discusses how perceptions about newcomers from service providers and organizations, often embedded in racial and colonial discourse, can negatively influence newcomers' access to employment and their interactions with the labour market.

\section{Cognizance of multiple identities and issues}

Assigning politicized narratives to individuals based on one of their identities can make newcomers feel unwelcome, solidify differences, and does not address the needs of individuals with intersectional identities. For example, a study from the UK on the experience of LGBT asylum seekers exemplifies the importance of organizations to be inclusive, account for multiple identities, and refrain from focusing on a single identity/issue. The report indicates that LGBT asylum seekers have had adverse experiences with mainstream LGBT organizations, sometimes because they do not have the finances to support this group or because of antagonizing and racist attitudes from the organizations (Refugee Support and Metropolitan Trust, 2009, p. 64). In fact, Refugee Support and Metropolitan Trust (2009) discuss how organizations that are multi-issue focused and work with LGBT, asylum, or ethnic identity issues have provided adequate support, but are hard to find and access because they are primarily located in bigger cities such as London. 
This research is also applicable to services provided in newcomer youth programs. To implement and sustain an intersectional approach, newcomer organizations need to be aware of the intersectional support youth require, given that their identities are often, "ambiguous, flexible, and multiple" (Caxaj \& Berman, 2010, p. 27). Caxaj and Berman (2010) also emphasize the need for organizations that will cater and support diverse newcomer youth by stating that in public spaces or when familial support was not available, newcomer youth expressed the need for support and safe spaces where they could show their authentic, multifaceted personalities (p. 26). While there are various ways to incorporate an intersectional lens and approach to a newcomer youth organization, researchers agree that it is not simply enough to name race, class, gender, and sexual or further oppressions etc. because it might reproduce feelings of otherness and existing forms of oppressions (Yee et al., 2014, p. 90). Instead to be truly effective, community-based organizations must educate themselves about issues pertaining to those identities (Ontario Healthy Communities Coalition, 2004, p. 11) and practice working to eliminate barriers.

Furthermore, research also sheds lights on the significance of acknowledging factors of multiple identities and issues when providing services to disabled newcomer youth. The lack of comprehensive services that recognize intersectionality for disabled newcomer youth and their families affect the quality of service they receive from community organizations. King et al. (2011) highlight that newcomer families with disabled youth have higher levels of unmet needs than non-newcomer families, specifically when looking for services, advocacy, and day to day support for youth (p. 2). Per Dix et al. (2009), inadequate support can be alluded to a lack of knowledge about newcomer families raising disabled youth who might require family-centered or culturally sensitive care. King et al. (2011) stress the important of culturally sensitive care, 
which is defined as approaches, attitudes, and behaviors of service providers that recognize the multiplicity of identities and factors, and is often used in childhood disability literature. To address the holistic needs of diverse newcomer families and disabled newcomer youth that have multiple intersecting identities and issues, "respectful and supportive treatment, information provision, and individualized care that consider the beliefs, worldviews, and preferences of families" is needed (King et al., 2011, p. 11). King et. al (2011) also discuss that organizations' and service providers' gaps in knowledge of the influence that ethnicity and settlement status can have, could result in them being unable to provide multi-issue service needs and outcomes (p. 11).

\section{Need for more comprehensive and cooperative service provisions}

Van Ngo (2009), advocates for the collaboration and codependence of service providers throughout multiple sectors that work with immigrant youth since their involvement and understanding of newcomer needs is more insightful once it is holistic. He adds that settlement agencies and mainstream organizations should aim to develop partnerships supporting newcomer youth to deal with, "diverse, complex developmental and acculturative issues, and promote their equitable participation in the community" (Van Ngo, 2009, p. 94). A similar sentiment is expressed by Yi et al. (2016) who highlight that because many newcomer youth experience various intersecting issues and inequalities such as poverty, discrimination, abuse, stigma etc., there is a critical need for an integrated approach that supports those individual needs and simultaneously promotes equality (p. 1083). Addressing newcomer youth needs also requires services that collaborate with schools, community organizations, and government organizations when developing programs so workers can utilize each sectors' specialty and strengths (Yi et al., 2016, p. 1083) to provide the most comprehensive service that caters to all aspects of newcomer 
youth identities. Furthermore, Travers et al. (2010) also advocate for similar collaborative and holistic initiatives by criticizing single issue/specialized organizations as being "outdated and ineffective for meeting the needs of an increasingly large number of very vulnerable youth with broad needs" (p. 195). Instead, systematic structural change is recommended to youth agencies so that no one aspect of youth needs is dismissed or ignored, and instead organizations feel the responsibility to respond and serve all youth (Travers et al. 2010, p. 195).

The role of service providers and workers

Amongst the significant impact that newcomer youth agencies and organizations can have during resettlement, research also emphasizes the influence and crucial service of frontline workers and staff members in those programs. The role of workers and representatives in such newcomer agencies cannot be understated; their strength and knowledge lies in being able to serve and empower marginalized communities, while also advocating for change and improvement based on their direct interactions and experiences with the clientele they serve.

Some potential outcomes of service professionals and workers interacting with the newcomer population are outlined by Gayton et al. (2007), who state that mentors can act as guides for families trying to overcome resettlement barriers and to youth who are adjusting to new structures and lifestyles (p.12). Furthermore, the article suggests that service providers and counselors can diminish new cultural or generational barriers that occur between children and parents once newcomer families begin to adjust and adapt to a new country at different rates (Gayton et al., 2007, p. 12). Service providers have the potential to support, represent, and change circumstances for newcomers once they arrive. To do so effectively, researchers suggest that they must actively engage in anti-oppressive practices that pay attention to "the ways in which particular forms of intersectionality inform everyday realities" (Brotman \& Lee, 2011, p. 
154). Therefore, service providers must maximize their scope and capabilities when dealing with newcomers, and newcomer youth specifically. Cognizance of intersectional identities is vital.

For example, Travers et al. (2010) suggest that despite the increased visibility of transgender people in society, service providers struggle meeting their needs because of misinformation and a "lack of evidence-based information to guide them" (Travers et al., 2010, p. 196). While service providers are more equipped to help LGBTQ youth with "coming out", there are pressing issues for transgender newcomer youth that they might not be prepared to help with, such as, the relationship between health and well-being, settlement issues, complex intersections of new vs. old cultures, and the discriminatory attitudes youth might face (Daley et al., 2008, p. 21). Bullying of transgender newcomer youth is particularly severe in both their home and host countries, and in ways that Canadian youth or queer youth might not face (Daley et al., 2008, p. 21). This is due to the intersections between racism, xenophobia, classism and homophobia, which is often heightened when mixed with their newcomer status and the "public" nature of transitioning (Daley et al., 2008, p. 21). In addition to all of this, the role and significance of service providers and frontline workers is magnified by Lee and Brotman (2013) who add that providing services to queer and transgender youth requires a development of "the historical, social, political and structural dimensions that shape the complex ways in which multiple oppressions operate in their lives" (p. 171).

\section{Service providers as resources for insights and critiques}

Research on the specific ways service providers can contribute to the advancement of newcomer youth program services and functions indicates that they can provide the most resourceful and nuanced perspective. For example, lack of knowledge training, difficulty assessing client needs, and language/cultural barriers were all identified as parts of service 
delivery that service providers claimed limited their reach and quality of service for newcomer youth (Yi et al., 2016, p. 1082-83). Furthermore, service providers identify that hostile public sentiment on immigrants in South Korea, who are largely perceived as a group that don't deserve social services, contributed to the difficulty when trying to support immigrants who faced a stigma and felt unwelcomed (Yi et al., 2016, p. 1082-83).

Moreover, Travers' et al. (2010) case study allows service providers to share their insights and experience about policies/practices on the provision of sexual health-related services for youth in Toronto. Focus group data shows service providers expressing the need for more ongoing staff training pertaining to LGBT youth issues, which would be one method to influence institutional policy changes on the way that LGBT youth with intersecting identities are served (Travers et al., 2010, p. 195). Lee and Brotman (2013) identified that there is scarcity in practice and research on how to actively engage in anti-oppressive social work and community organization with queer and transgender youth in Canada (p. 170). A similar sentiment is expressed by Travers et al. (2010) with regards to service provisions for transgender newcomer youth. Van der Meulen et al. (2010) also use the response and participation of service providers in their study about sexual health services for newcomer youth. They conclude that service providers can identify core issues/concerns of newcomer youth programs in Toronto, especially as they pertain to specific groups of marginalized youth including, Black youth, newcomer youth, youth with disabilities etc. (Van der Meulen et al., 2010, p. 188). Service providers also highlight that they lack funding, support, and resources to target the needs of each group, despite acknowledging that each require significant individual and collective support and they often have to rely on the help of volunteers to carry out initiatives and programs (Van der Meulen et al., 2010, p. 188). The service providers also discuss extreme barriers to service provision such as 
poor resources for language translation of sexual health information in a diverse and multilingual city like Toronto (Van der Meulen et al., 2010, p. 188).

Barriers when supporting and providing services for newcomer youth

Issues that newcomer youth face are often magnified because of their intersecting identities and they can feel marginalized/excluded from service provision. The following section outlines some barriers, limitations, and obstacles faced by newcomer youth with multiple intersecting identities, and the difficulties they have when trying to receive support and services from newcomer youth agencies and programs. More specifically, this section highlights issues and discrepancies pertaining to homelessness and mental health support, which are emphasized and heightened for newcomer youth with multiple identities. Case studies and examples examine how newcomer youth might feel distanced or discounted from society, certain programs, and service providers because there is a lack of understanding of their unique, complex, and intersecting identities.

\section{Homelessness}

An article by Berman et al. (2009) examines the connection between dislocation and homelessness faced by newcomer and Indigenous girls and the effects they have on their wellbeing. Identifying that there is a lack of literature on the intersecting influences of emotional well-being, homelessness, and forms of oppression such as poverty, violence, and racism in girls' lives (Berman et al., 2009, p. 419), the authors discuss the barriers faced by racialized newcomer girls when facing homelessness, claiming that their intersecting vulnerabilities make them feel devalued and that they struggle to find belonging (p. 420). The feelings and experience of "otherness" that uprooted and/or displaced young and racialized girls have while occupying 
liminal spaces, are in part due to their identities of race, gender, socio-economic status etc. (Berman et al., 2009, p. 422). In fact, the article concludes that while individual and personal circumstances play a role in characterizing these young girls' experiences with homelessness, the amalgamation of those factors and the situations of newcomer and Indigenous girls who are displaced reflect their vulnerable, subordinate, and unequal positions in society (Berman et al., 2009, p. 427).

Similarly, a 2014 study by the Centre of Addiction and Mental Health (CAMH) on newcomer youth homelessness highlights that the newcomer youth population in Toronto is immensely underserved, yet very vulnerable to homelessness and discrimination. When discussing systematic discrimination, newcomer youth participants in the study identify that they often felt they received inequitable treatment from service providers based on "age, gender, race, sexual orientation, mental health needs, status as a newcomer, status in Canada, and being a young parent" (CAMH, 2014, p. 16-17). In addition to the unique hardships and challenges homeless newcomer youth face prior to their migration to Canada, they also all come from differing backgrounds and associate with multiple identity groups (CAMH, 2014, p. 27). Researchers expressed that many newcomer homeless youth struggle having to mediate and decide which of their identities takes priority when attempting to seek help from service providers (CAMH, 2014, p. 27). Similarly, homeless newcomer youth also communicated that they fear a hierarchy exists within the service system where newer and more recently arrived youth receive priority when accessing services and housing, instead of others who are unsettled and have been in Canada longer (CAMH, 2014, p. 27). 


\section{Mental health support}

Furthermore, research alludes to the ways that access to adequate mental health services for newcomer families and youth is intertwined with intersectionality. Khanlou and Crawford (2006) claim that the intersections of gender, life stage, migrant status, and race play a significant role for newcomers and require intensive mental health promotion, education, and practice in a multicultural setting (Khanlou \& Crawford, 2006, p. 54). Similarly, Khanlou et al. (2009), advocate for strategies which consider multiple dimensions of mental health and wellbeing, for example, individual circumstances, resettlement, employment, language, etc. (p. 32).

Specifically, they mention the interplay of language barriers, socio-economic status, and cultural differences as challenges that newcomer youth face when dealing with mental health services. Access to community based mental health services for newcomer youth is hindered by linguistic barriers and poverty. Khanlou et al. (2009) explain that there is a lack of mental health services in areas where newcomers reside, such as the suburbs, due to their inability to afford the high cost of living in the downtown Toronto area (p. 31). Additionally, the strenuous or expensive transportation routes required to get to the downtown locations makes it virtually impossible for newcomers to easily access mental health services (Khanlou et al., 2009, p. 31). Lastly, Khanlou et al. (2009) acknowledge that comprehending cultural contexts of newcomers from diverse backgrounds is imperative because often the Western understanding of mental health, which is embedded in a separation of mind and body, is very individualistic and might not resonate with newcomers' comprehension of mental health support and services (Khanlou et al., 2009, p. 30$31)$.

Cohen (2016) examines why refugee youth are more likely to suffer adversities and mental health issues, yet are the least likely to seek mental health services (p. 133). Lack of 
awareness of available mental health services or the inability to navigate the new systems in the country partly explain the low participation of refugee youth in mental health services (Cohen, 2016, p. 96). Additionally, the fear of being stigmatized or further marginalized for mental health problems, along with parents underestimating stress or mental health concerns of their children is also noted by Cohen (2016) as potential contributing factors (p. 97). Furthermore, she acknowledges, much like Khanlou et al. (2009), that some of this disconnect can be assigned to the Western system and understanding of mental health which newcomers fear might not be sensitive or equipped to cater to their cultural beliefs, needs, or symptoms (Cohen, 2016, p. 133). Moreover, the idea that language and accessibility barriers can contribute to a lack of seeking mental health support and services is also reaffirmed in this study (Cohen, 2016, p. 133). 


\section{$\underline{\text { Research Questions }}$}

Based on the review of literature on intersectionality theories, practices, and identities, this research study poses three primary research questions:

1) How is intersectionality understood by staff and frontline workers in newcomer youth organizations?

2) What are the experiences that service providers have with newcomer youth who have intersectional identities?

3) How is intersectionality applied, incorporated, and promoted in newcomer youth programs in Toronto?

Furthermore, one of the main goals of this research is to explore how and why an intersectional lens and approach is beneficial in the implementation of newcomer youth programs in Toronto. I would like to examine how being cognizant of intersectional identities in community organizations is a useful tool to facilitate an understanding of the unique and complex sociopolitical sites encountered by newcomer youth. Lastly, I am looking to learn more about best practices to support newcomer youth that incorporate a practical application of intersectionality. 


\section{Methodology}

\section{$\underline{\text { Research design }}$}

To better understand newcomer youth programs and their engagement with intersectionality, a qualitative research design is utilized in this study. Qualitative research is embedded in different forms of data collection, such as interviews, focus groups, case studies, etc., and this study uses the method of in-depth interviews with key informants to garner results. Creswell (2012) emphasizes qualitative research as a process, beginning with a theoretical framework which pertains to a problem or research interest on individual, group, human, and social problems (p. 44). He adds the next steps to the process as; the collection of data in settings that adhere to the people and location the study is about; inductive and deductive analysis of the data to extrapolate patters and trends; a representation of participants' voices; and researcher's reflections interpretations, and descriptions in a report or presentation that contributes to the existing literature (Creswell, 2012, p. 44). Creswell (2012) also establishes when it is appropriate to use qualitative research, stating that this method is most useful when there is a need to study a group, population, hear silenced voices, gather a complex understanding of an issue, and empower individuals (p. 48). Furthermore, he claims that quantitative measures and statistics do not study interactions or uniqueness of people while being sensitive to gender, race, and economic differences in the way that qualitative studies do (Creswell, 2012, p. 48). This description and reasoning for using qualitative data align strongly with the intentions of this study to discuss and infer the intersectional approach of newcomer youth programs in Toronto.

\section{$\underline{\text { Sampling design }}$}

This research study uses purposeful and non-random sampling to recruit participants (Creswell, 2012). Interviews in this study were conducted with service providers in newcomer 
youth organizations in Toronto, who are identified as key informants in this research. The findings in this paper are grounded in the information and revelations that key informants provide about the application and cognizance of an intersectional lens in their workplace programs, as well as their experience providing support and assistance to newcomer youth who have intersectional identities.

Community organizations and agencies that provide newcomer youth programs in Toronto were contacted and interviews of 3 service providers were conducted. Participants in this study were interviewed if they met the following criteria: i) a current social worker or service provider at a newcomer youth program or agency; ii) someone who has experience dealing one-on-one with newcomer youth with diverse backgrounds for at least one year in their current workplace; and iii) someone with knowledge and understanding of the programs and services their workplace offers to newcomer youth. The reasoning and significance for using key informants, such as service providers and frontline workers, is because they are not only experts that have a plethora of experience to draw from, but because they are also representatives for specific programs and agencies in Toronto and are the most equipped to discuss their services indepth. Hughes and Preski, (1997) allude to using key informants as a way of relying on a small group of knowledgeable participants who can describe and articulate social relationships (p. 82). They add that the data key informants provide are based on their; "unique access to organizational information or specialized knowledge about group or organizational properties or events" (Hughes \& Preski, 1997, p. 82). Like this research study, Agyekum and Newbold (2016) also used in-depth interviews with key informants to gain revelations about newcomer youths' sense of belonging and mental health in Hamilton, Ontario. Their study similarly reached out to 
service providers through purposive sampling and gathered meaningful responses and connections in their findings.

Data collection and analysis

The recruitment process was done by posting recruitment posters (See Appendix B) at specified agencies, as well as emails/ phone calls (See Appendix C) to service providers and managers who could pass along the information to their colleagues. When participants expressed interest, they received more information about the study and were sent the consent form which emphasized the voluntary and confidential nature of this study. Once they had the time to read, reflect, and sign the consent form, a mutually convenient time and place was set to conduct interviews. The interviews were conducted in private meeting rooms at the participants' workplaces. Interviews lasted about 60-75 minutes and were audio taped to facilitate analysis, which participants had agreed to in the consent form. Interviews entailed semi-structured, open ended questions that were not tailored to yield any specific responses, but more so to generate a conversation about the participants' understanding, experiences, and insights on intersectionality in newcomer youth programs. To facilitate a discussion, the interview questions (See Appendix D) were structured to discuss: i) the participants understanding of intersectionality as it pertains to newcomer youth; ii) how and whether cognizance of intersectionality shapes the way participants can provides services to this population; iii) how an intersectional lens is applied to programs in their agency/organizations; and iv) specific instances and anecdotes participants had about newcomer youth who have intersectional identities.

Once the interviews were transcribed, they were organized and coded to reflect the insights related to the research questions. As proposed by Neuman (2014), this step involved creating thematic categories and tagging the data with codes that guided and emerged in the 
interviews. This made it easier to organize the data to find common themes and then use thematic analysis to identify larger and overarching concepts that emerged in the study (Neuman, 2014, p. 79). The conversations from the interviews were combined with field notes, the coded analysis from the transcriptions, and the themes and frameworks from the literature review to allow for a triangulation approach to be taken in this study. Triangulation is defined as the result of collecting data from "multiple sources, through multiple methods, and at multiple times—and analyzing the data from multiple theoretical frameworks" (Neuman, 2014, p. 84). This is done to generate findings which are comprehensive and representative of a variety of perspectives on a certain topic or issue (Neuman, 2014, p. 84), such as intersectionality and newcomer youth services in Toronto for this research. Moreover, due to the limited number of participants in this study, generalizations about all newcomer youth programs or their application and recognition of intersectionality should not be made. However, given that the number of newcomer youth that each agency provides services to is relatively high, it is worth mentioning that collectively, the programs and services discussed in the data potentially reach and serve a large number of newcomer youth in Toronto, and the findings are, thus, noteworthy insights toward future largerscale research. 


\section{Findings}

The following section outlines key informants' responses and recurring themes that were discovered through the interviews. Findings are divided into three sections, intersectionality, barriers when accessing services, and organizational approaches to intersectionality, with subsections focusing on more detailed patterns and themes.

Interviews were transcribed and coded to reflect the research questions in this paper, and direct quotations are used to highlight key informants' ideas. Participant responses are presented verbatim and thus include their exact phrasing, grammar, and word choices. Identifying information such as names of newcomer youth agencies/programs are not stated to avoid disclosing more detailed insights about such organizations. Pseudonyms are used for the names of participants to maintain anonymity; however, their job title is identified to provide context and help ground their experiences based on their type and level of interaction with newcomer youth in Toronto.

The three participants in this research study have worked at their respective youth organizations for a minimum of one year. Two participants, Janice and Yesenia, are females and one, Frank, identifies as male. Janice is a Newcomer Youth Settlement Worker, Yesenia is the Team Leader at a Newcomer Youth Program, and Frank is a Case Lead Worker. When asked how often and to what extent participants interacted with newcomer youth, all responded that they saw newcomer youth on a regular and consistent basis, despite the nature of their interactions varying greatly. Participants defined newcomer youth as individuals aged 13-24 who are immigrants, refugee claimants, or have refugee status, have been in Canada for less than five years, and are looking for support to settle and integrate in Canada. This is because their services and programs are generally catered to those who are teenagers or older. 
Intersectionality

This section maps key informants' understanding of intersectionality, how intersectionality influences the way they provide services and support to newcomer youth, and lastly how they perceive having multiple intersecting identities shapes the settlement experience of newcomer youth.

\section{Participants' definition of intersectionality}

When asked how they would define intersectionality and what it means to them, participants gave varying answers. They used examples and drew upon personal experiences with newcomer youth to exemplify how they would define the term. Janice thought intersectionality was being aware of an individual's multiple identities and using that awareness to provide people with accommodations. She described it as such:

$\mathrm{J}$ : It's being aware of someone's multiple identities, varying statuses in life, things that they've been going through, and being able to accommodate that into the programs that we do. It's, I think, intersectionality is the basis of why we're being accommodating in our program, it's because we know that a lot of the youth that comes through our space comes through different backgrounds, different experiences, and know different struggles.

Frank viewed intersectionality as a tool that recognizes identities can be a baggage and that lack

of acceptance of certain identities can lead to difficulties for newcomers. He put it like this:

F: Well, intersectionality can be that newcomers are here they are coming with their own identity. Whether they come as Muslims, or African Americans, their identity is itself baggage. And when they arrive, most of them are not accepted the way they should have been accepted. So, these barriers come in and they're having many difficulties together.

Yesenia believed intersectionality was when multiple identities come together and determine how people identify themselves, the paths they take in life, and their access to certain things. 
Y: I think intersectionality is when all these identities, ethnicity, culture, socioeconomic [status], gender, I believe it's when they all come together. I think it really shapes how a person identifies themselves and how they see this world, it eventually kind of determines their paths and what kind of programs they want to access. It's you know if they feel included, if all the factors are there, if the programs are acknowledging that you know, newcomer youth come in with different experiences.

\section{Personal approaches to service provision}

Below is a summary of how key informants believed their personal method and approach to providing services for newcomer youth is informed by an intersectional lens. More specifically, this entails how they think their earlier definition of intersectionality, and the awareness it entails, is incorporated in their interactions with newcomer youth. Frank attributed intersectionality for making him and his organization more accepting, and ultimately making it easier for newcomer youth to feel welcomed:

F: Because of our understanding, we are aware, we make it very open to them, we listen to them, and we won't turn on and say anything but that we accept them. Whether it is, be their depression, whether it is any mental health issues, whether it is the drinking habits. We make it easier for them to open up.

Frank also shared an instance he claims made him more aware of the different identities youth have and how it can result in various levels of marginalization. He explained that he accompanies youth who have gotten in trouble with the law, to court hearings. Frank believes that racialized youth often feel uncomfortable in court for multiple reasons, and after accidently addressing a transgender racialized youth with the wrong pronoun, he noticed they felt even more marginalized.

F: I work with diverse youth, and in the courts, we go by the last name, so I'll be calling you Mr./Ms. instead of your first name. Because of my age, and I have seen the young people feeling odd about it — because we are in an already hard situation, and I have seen you know, people who are transgender and I am accepting them, but I will by accident say incorrect pronouns. And I have seen you know, it makes them more uncomfortable, they are already in disadvantage at court. I feel bad I am more careful now, I always ask what they think first before I say. 
Janice shared that intersectionality directly changes the way she does programming as it

makes her more flexible and innovative. Through frontline work with newcomer youth, she can apply and evolve her theoretical framework of intersectionality to be more practical. She put it like this:

$\mathrm{J}$ : You need to actually work in the field to understand these things, it [intersectionality] changed and impacted the way I do programming, the way I come up with new ideas, new programs, right? At school whenever I would do workshops for projects I always do it the standard way, but now thinking of intersectionality makes me more flexible, more innovative, makes me think of better ways to accommodate youth in workshops. Being able to know theories and actually understanding it, helps me apply it to the workplace.

She also thought that without intersectionality the value of their programs would diminish and the focus would solely be on statistics and numbers:

J: If I didn't know about intersectionality, I'm only going to be focusing on getting as much newcomers I can into the programs. Then that doesn't really put value into the program at all, into what we do in the program at all. Not really reconstructing our programs every now and then, able to accommodate every need the youth has. So, it's going to be an easy work, I tell you, if I don't think about intersectionality.

Similarly, Yesenia mentioned that it makes her organization more responsible and critical to ensure that accessibility issues and barriers are addressed. She also felt that understanding these needs makes her more passionate:

Y: I think we're always analyzing those things, for example, when we take into account like, a girl's leadership program that's running right now. Not everyone has the same needs, the same learning phase. We look at the different barriers and accessibilities that newcomer girls have, that the reason that program even came about was because there was no program in the neighbourhood for girls. It's hard, I came from a background of very similar experiences and I can identify. It makes me more passionate, if that makes sense.

\section{Settlement experiences of newcomer youth}

Participants also provided information as to how they thought having multiple intersecting identities shapes the settlement experience of newcomer youth. Key informants' understanding of the way that intersecting identities work directly influences their application of 
intersectional approaches. Yesenia gave a few examples that she's noticed since working with

newcomer youth:

Y: From what I've seen, they often experience, like a cultural clash between what they pick vs. their parents. Parents have a lot of expectations on how their identities should be, whether that be ethnic or cultural, what their practices should be. They [newcomer youth] are already trying to settle in a new country and most, I would say $95 \%$ of our kids are in high school so they're between the ages of 14-19, already being thrown in a new school system and making new friends is hard enough, they have all these expectations laid by their parents. Because that's also the age their exploring their own identities, as a newcomer who's only in Toronto for less than year or two years, you're already facing a clash between what's at home, what you want to identity as, and what peer group you want to be involved in.

Likewise, Janice also identified confusion and identity conflicts as something that impacts immigrant youths' experiences. In fact, Janice cites that the domination of western culture in some of their homeland countries makes it harder for youth to hold on to their roots once they come here, which then causes tensions and conflictions:

$\mathrm{J}$ : In terms of identity, there's this thing in certain countries where the Western culture is pretty dominant, it's seen as dominant over their own culture. So, when youth come here it's hard for them to hold onto their roots. Whatever's hip in the Western culture is hip back home, when they come here, although it's easy for them to adjust but hard for them to hold on to the culture they used to have, which confuses their identities, sense of belonging, and their parents too.

Janice said she understood the position that newcomer youth are in, having once been a newcomer herself, but shared that they are not always very vocal about the settlement and adjustment issues they have. She said:

J: I mean, I'm a newcomer myself, and I see that many of the youth, they're always, it's not always, but there's this feeling of confusion almost. Whenever they come in, you know, there's the piece that they should adjust to their new community and there's that piece where they're only 15 , they're only 16 , they're trying to figure out who they are. And it's really, I find it conflicting for them? And, I mean, they don't, you know, they're not vocal about it, a lot of the youth that we work with, they're not vocal about it.

Frank thought that having multiple intersecting identities was a direct reflection of the settlement barriers and limitations that certain identities carry. He stated that newcomer youth struggle 
because they feel pressured to change themselves to adjust and integrate. He added that the dominant society should accept and welcome them before they feel this way.

F: Their [newcomer youth] accesses would be limited over ones who have been here, what I would say, the norm dominant society. They might not be knowing it or you know, the people who are providing the services may have biases. So, they are into the loss. Youth who are coming here, they cannot change themselves, they should be able to be proud and say that this is the way that I am and we should be able to accept them the way they are. Rather than having difficulty or you know, trying to forcing people into accepting them. Show them before this that even though you may be a queer, you may be a black, you may be a Muslim, it doesn't matter; our attitudes, our behavior, should show that we accept them.

\section{Barriers when accessing services}

The following sections outline barriers and limitations that newcomer youth with intersecting identities have when accessing programs and services. First, discussions of "youth spaces" is highlighted to emphasize patterns, acts of exclusions, and discrimination, some amongst newcomers themselves, which participants thought was imperative to share since it determines newcomer youth willingness to participate. Secondly, key informants discuss their perception of what specific identity groups face hardships when trying to participate in their programs and services. Specifically, newcomer youth from lower socio-economic statuses, LGTBQ youth, and those with mental health issues are mentioned. Lastly, personal anecdotes from participants are revealed to show how multiple identity groups can intersect and overlap to create further barriers and limitation for newcomer youth.

\section{Youth space}

While Janice and Frank shared that the youth space in their organizations were generally accessible and popular amongst newcomers, Yesenia thought theirs was inaccessible for multiple reasons. She explained it as such: 
Y: Okay so I don't think our newcomer youth base is very accessible. Yeah, because it's on the second floor of this building and unless we tell youth, this is where we are, some youth already have a hard time navigating the TTC, and then you want to tell them [address removed], and they're like ok where is it? And you have to coordinate and bring them and go through all the other staff members, it poses a challenge. So, I think, as a space, I'm cautious because I understand funding, but it's a youth space, we should be more street front and more accessible, and people need to know more about it, so it's not that it's a hidden gem.

She added that she'd like to see it transformed:

Y: I think that's a dream for me in five years, if they can move this to a nice community center or like, even a street front kind of place, that would be nice.

Another reason she deemed it inaccessible was because of its lack of acknowledgment of

LGBTQ newcomer youth identities. She was critical of the lack of posters and signs that would

make LGTBQ newcomer youth feel accepted and welcomed. Yesenia described it as the

following:

Y: Yes, the physical space is a first, and as an organization we're trying to do more of because that's the first thing the youth come to see. One of the things I'm working on with my team is exploring ways... see we're very focused on the newcomer culture identities that we're not exploring socioeconomic, and sexuality. So, what do we do with newcomer youth who identify as LGBTQ? Is our space in anyway showing that we welcome those types of youth? And we don't, we don't have any of those signs, basic stuff that the City of Toronto would have, we don't have that physical element, we don't have it in our space. That's something we talked in length about, and we are trying to put to action by September to have that physical aspect in our space.

\section{Dynamics between newcomer youth}

The youth spaces prove to be resourceful to not only newcomer youth, but also to the key informants. Key informants highlighted patterns and instances they notice in the youth spaces that they thought hindered the inclusion of all newcomer youth. In addition to the physical space not recognizing LGBTQ identities, as outlined by Yesenia, tensions and cultural clashes amongst newcomer youth can make the youth space uninviting for some newcomers. For example, Janice overheard newcomer youth expressing their disdain for the youth space because they felt it was overshadowed by one group only: 
J: I think one thing that just popped out to me is the way youth perceive other cultures. Something that the youth think is, oh this group has always been in [the] space, has always had some ownership into the program. So, next time they're invited into programs we hear them ask, "Oh are all the other Filipino youth going to be there?" Right? Those sort of things, sort of like aggression, yeah sort of micro-aggression happening there.

She added that there was also visible hostility between two specific ethnic groups:

J: We have a lot of Tibetan youth as well. You know, that's something that we have always been cautious about when they interact with Chinese youth. It's because of the whole history, tension of the countries.

Yesenia also drew upon a similar situation she noticed when there was a mixed setting between

more newly arrived newcomers and those who have been here longer.

Y: The really new...new newcomers don't feel comfortable and they're experiences are different. Like if this club is overtaken by one specific ethnicity and they don't identify with that, they don't feel comfortable continuing. What happens with newcomers who identify with one specific ethnicity is they tend to communicate within their language... and we have one or two youth who are not in the same ethnic group, and don't speak that language. No matter how much you want to bring them together, they feel a little isolation.

However, Yesenia did add that this was not always the case and praised newcomer youth for their ability to embrace and welcome each other. She said that while situations she outlined above might occur, newcomer youth from different backgrounds can become friends over their shared newcomer identity:

Y: What I find about newcomer youth when I'm working with them is that when they know there's another newcomer youth with them they're so good at embracing and welcoming them. We see a lot of that where there's newcomers and they don't have any friends and then they build this relationship, and they'll have friends after the program and they'll set up times to meet on weekends and stuff like that.

\section{Discussing oppression and discrimination with newcomer youth}

To mitigate obstacles that might arise from contentious relationships or situations

between newcomer youth, participants felt the same obligations to intervene. Frank discussed a program his organization is planning which encourages conversations amongst newcomer youth and could eliminate such misunderstandings. He explained: 
F: As a matter of fact, right now, before every month we are having a program. Usually they [youth] would come and see me once a month. So, what we have changed now, that we will be sitting with everybody, all of them, we will not be segregating them. They come and sit down and talk about anything that the youth wanted to talk about. That will be taken on in September. This way they will be able to turn on and speak with us and each other.

When speaking specifically about hostility between identity groups, he suggested that an open conversation and letting newcomer youth discuss their biases amongst themselves could result in a progressive engagement.

F: Yes, so those that are not accepting, they will be able to turn and say for example, "oh if that individual happens to be gay, we don't have to accept him or her" So, we let them speak, because by speaking to them, speaking to each other it's easy [rather than] without knowing who or what identities are there. Individuals may be participating, but this will be the awareness, this will be making decisions by engaging into with, not, I don't call it argument, but conversations. Let them speak up and say this is what I believe, let someone turn around and say "why do you believe it" "well I believe it because whatever the reason..." That individual may be able to learn from the others perspective.

In contrast, Janice was uncertain how she felt about having conversations of such nature in opensettings, and instead shared that she and other staff prefer speaking to newcomer youth alone and directly:

J: I think, we've always been trying to talk to the youth alone whenever we hear some discriminatory stuff from them, any aggression, anything small, we always try to make them understand, talk to them right away, tell them that they're not supposed to be saying that sort of things.

When trying to think of other ways staff could address such situations which make some newcomer youth feel unwelcomed or uncomfortable, Janice contemplated having antidiscrimination and oppression workshops, but wasn't confident whether they would be feasible or useful. She said:

$\mathrm{J}$ : But I think in terms of that, we have a lot of youth in that capacity that it's almost hard, almost like, so many different understandings of oppression, it's such a diverse set of clients. It's almost something we can't control. The staff is like are we supposed to create a workshop about this? Are we supposed to do something about this? But then, are youth going to be interested in this? There's that whole new other piece of how much can we put this word out there? 


\section{Identity-based limitations for newcomer youth}

Throughout the interviews, participants gave many examples as to how youth with certain identities face additional complications when accessing programs. Outlined below are discussions of specific identity groups key informants believe go through various obstacles when seeking support and services from organizations.

\section{Socio-economic status}

Lower socio-economic status was identified as a considerable limitation and hindrance

for newcomer youth participation in programs. Yesenia put it like this:

Y: Ok so one example is that their [newcomer youth] parents are working, both their parents are working two, three jobs, and we're running an after-school program. They [newcomer youth] need to be at a different public school to pick up all their siblings, because their parents can't and they need to go home and babysit them because they don't really see their parents. They have to go cook, and be responsible. So, understanding their socio-economic status, what kind of support they need to access, from the beginning, and being as accommodating as possible to those type of youth is, I think that's number one for me.

Janice mentioned that socio-economic status was something they always consider when doing

programming because, coupled with family dynamics, not having the resources or time to

participate because of financial hardships is very evident. She described it as such:

J: Socio-economic status and family dynamics are the main identities that can dictate access and experience in our newcomer youth programs. Like whenever we have programs, you always have to think of youth not having the money for TTC, so I always offer tokens. Or, you know, even if youth are very new to the country, their parents don't have the time to help them navigate because they're at work, so even if it takes for us picking them up from the subways station, their first time, just going there.

On the same note, Frank cited financial stress and responsibilities, stemming from lower socioeconomic statuses, as a reason why drop-in programs are the most popular and accessible for newcomer youth. 
F: In drop in time, the youth are able to come in and out. Many have restrictions, jobs, responsibilities at home. Fixed program times are not accessible. Whenever they wanted to come, they come. Some of them come for music, some of them just want to sit and chat and play games. Some they want to engage with the school homework. If their friends are there, just being with them. They have financial stress many of them, so they can come here to relax anytime.

\section{$L G B T Q$}

When talking about LGBTQ youth, both Yesenia and Janice said their organizations want to be able to address their concerns and needs but find it difficult to talk about, mainly because they don't see enough newcomer youth who are "out". Janice put it like this:

$\mathrm{J}$ : In terms of gender and sexuality, we've always been exploring, there's always been ideas about programming, tackling LGBTQ issues but we just, we don't see a lot of youth who've actually come out and who are really open about their sexuality. You know, the way that you know, other places might be. So, that's something we kind of are a little bit on and off. Because we don't know, right? If you are not ready, then why push it? That's the main thing about that.

Yesenia had similar concerns and identified that her organization is working hard to address

these discrepancies. She detailed an unsuccessful attempt at taking the newcomer youth to the

Pride Parade to stimulate a healthy conversation around LGBTQ issues.

Y: Even for the pride parade, I was a huge advocate on taking a couple of youth as a first step to show that we are trying to be inclusive of youth who identify as that. It didn't work out because our management said there's a lot of nudity so we don't want to take [the] youth. Which I do understand, but I also thought it would be a great social event to kind of open that subject with youth instead of a workshop. Like we're going on a trip to the pride parade. If it's an informal social trip, these youths may be more comfortable and we can have that healthy conversation, right? Start it. That was unfortunate. But I'm sure we'll find a way soon.

\section{Mental health issues}

Janice had a similar take on how she's noticed mental health issues impact newcomer

youth access to programs and services. Due to the hidden nature of most mental illnesses, and the reliance on newcomer youth to disclose their mental health issues, she found herself in a helpless situation. Below, Janice describes when she realized youth with mental health concerns just need accommodation and support, but was unable to provide it to one newcomer youth: 
J: I started working with this youth when I first came here, when I first got hired, and she's always been distant. She would say that she wants to go to this program and next thing you know she's not showing up, and she started to not show for a few months. Recently she started bouncing back, she started coming to the program again, and accessing one on one services and I recently realized that she was diagnosed with schizophrenia. It made me feel bad, I was thinking she just doesn't want to come to the program anymore. We need to realize that for most part, it's not that simple for many youths. It's not that they don't want to go, they wanna go, but they need further accommodation, all they need to do is ask for it. You have to wait for them to disclose, but at the same time, they're very fragile and very careful of these things. I don't want to hear something bad happened and I could've done something about it. I'm always cautious especially working with youth with mental health problems. I've definitely been more open to the possibility of mental health issues in the past year that I've worked here. It's made me more appreciative of such services, made me enhance my skills set in terms of dealing with such issues.

\section{Personal anecdotes of multiple identities}

Key informants were adamant about sharing interactions and experiences with newcomer youth to support some of their ideas. Participants told stories that elaborated on the complexities newcomer youth with multiple identities face and how it influences the way they, and their organizations, support and provide services. The previous sections stressed how difficult it is for newcomer youth with one marginalized identity, and this section elaborates on the struggles that can arise when more than one marginalized identity intersect for newcomer youth. For example, key informants talked in-depth about how socio-economic factors and lack of cultural competency work together, along with the need for services catering to Muslim girls, and the tribulations faced by a racialized and homeless sex worker.

\section{Socio-economic status and the education system}

Yesenia mentioned that cultural background, family dynamics, and language are things she always considers when working with newcomer youth: 
Y: I really consider their cultural background and what kind of views and family settings they come from because that can really impact how often they can come to our program. Other factors would be language. I think as a settlement worker, working in this field it can get overwhelming when you're facilitating activities. Right now, I'm speaking really fast with you in English. When I'm with my youth, we have to be very careful because they might feel intimidated to say, Yesenia, slow down I can't follow.

She then discussed, in-depth, her experience with a newcomer youth who was going through multiple issues at once because of his socio-economic status, his family dynamics, cultural background, and lack of comprehension of the school system in Canada. In fact, it was so overwhelming, that at one point he started to develop anxiety and did not want to talk or seek help. Yesenia explained the situation as such:

Y: So, I had a youth that came to Canada a year ago, and he's a first-born child in his family so there's a lot of pressure from his parents about success. He's a senior, he was in grade 11 when he arrived, so there's a lot of financial pressure on his end, he has to work part time because his parents put that, "you have to be the golden child, and you're the oldest, you have to support" pressure. So, for him it's, ok university will cost so much, he's already overwhelmed with finances, he needs a part time job... so there's all these issues we had to work with. We did a lot of one on one sessions with him, he used to not come sometimes to the one on one session because he was like, it's too overwhelming, it was too much, I'm so anxious, my parents are never happy with me, things like that.

Specifically, lack of knowledge about school systems and the way post-secondary education works in Toronto were mentioned by Janice and Yesenia. They both expressed that there are knowledge gaps for newcomer families about school, and when mixed with financial and

familial pressures, it negatively impacts the way newcomer youth settle in Toronto.

$\mathrm{J}$ : A lot of newcomer youth have difficulty adjusting to the education system here in Canada. They don't know how course selection works in high school. Even parents, you can't blame the parents for not knowing what the system is. So, having a program that values education, and gives them specific skill set they could apply as they move on into their lives is something I really want to see now.

Yesenia referred to the newcomer youth mentioned earlier to exemplify the same notions and even criticized the schools for not adequately addressing the discrepancies and gaps: 
Y: He came into the school system when he was in grade 11 and they threw him into applied, or else he would have had much lower grades, and the parents didn't quite understand that. That you can't go to university with applied courses, and that if your son wanted to take academic courses then he would have started in grade 10. It was something that the school should have clarified with them. I think there's a barrier right there because the school system is not doing their job. We've been seeing a lot of that with our newcomers in schools because the parents don't get it. Then there's this sudden pressure from parents to kids because the parents don't know enough.

\section{Muslim girls}

Participants perceived Muslim girls as a group who are the most excluded and must overcome various barriers when trying to participate in the programs. Due to religious beliefs that frown upon programs with both boys and girls, or because some Muslim girls are not comfortable being expressive, playing sports, and engaging with males, it is difficult to sustain their participation. Frank shared that these ideas come from a very specific religious sect of Islam, which was for segregation amongst genders, and he noticed how it affects the participation levels of newcomer Muslim girls.

F: I would not hesitate to say it would be a Muslim youth who face barriers because of the music, and Muslim girls especially because of the you know, mixing up of boys and girls together. What I'm saying is that is what makes it harder for some people who are in that belief. I am aware of it, that there are some people who do not believe in that [co-ed programs]. Not all but some of the Muslims, you know, there's no believing in that stuff. Couple of the groups where there is a hardcore religious belief they would be having these barriers. They don't encourage the mixing of girls and boys in the programs, so many times Muslim girls will not attend or come to programs.

Despite being aware of how sensitive and personal these beliefs are, Frank thought that by educating parents and religious leaders about the programs, getting them involved in the programming, and having them see for themselves the impact, he might be able to change their perception. He attributed some of their hesitancy to the fear of not knowing exactly what happens during the programming, thus not allowing their kids to come. 
F: More education be given to those who are in belief of this, in having a segregation. In our organization, that I know, there's been a few. I live in this area and the group is a minority, so I try to talk to them the parents, the leaders. I think, if they can be sit together, and show them, we can make it work. Because at the end of the day, these youth, the girls, they can be deprived of the services because their leaders, or their parents don't believe in it. Rather they should be able to come in and see it, our programs, see ok there are other people who are engaging. They might be afraid just because they don't know what happens, or something they don't like will happen.

Yesenia criticized the lack of programs for newcomer girls in Toronto, but also

acknowledged the challenges that can arise when trying to curate such programming. She also identified how Muslim girls might feel excluded from programming, and shared ways that her organization tried to overcome that barrier. Specifically, she talks about one Muslim newcomer girl who expressed interest in the sports programs, but did not want to participate because she wasn't comfortable with the mixed nature of the program.

Y: The biggest barrier within this neighbourhood, or even in Toronto, is there's no program specific to girls. We started a girls sports group because there's no recreational activity that was open to girls and this came about because one of our youth who identified as Muslim really wanted to come out and play soccer and basketball but all our programs, like we did have a basketball and soccer program, but all of that was male dominated and you know, her religious background and her gender, it was a barrier. She said I'm wearing a hijab and I don't want to engage in sports activities while there's all these boys.

After creating a girls only recreational program, the organization found that parents did not want their daughters to be out after dark, yet the only free city permit to host these programs that the organization could get was in the evening.

Y: We thought about it and we were like, it's true there's no program in the neighbourhood that addresses this need, so we started the girls' sports group. But then the only permit [we] could get was a 6-8 pm school gym permit. For a bit, the girls did come out, but not just Muslim girls but a lot of the girls came out to the program. Another barrier was it was $8 \mathrm{pm}$ and in the Winter, it gets pretty dark outside and they can't be out that late, and then safety was an issue. Parents were not allowing them to come out so there was low attendance in that program. But we couldn't get an earlier permit because we needed a permit that was free so the school board was like, if you want a free permit, this is what we have. So, there we go, another barrier. 
To mediate obstacles that kept presenting themselves as the organization was trying to be inclusive of Muslim girls, Yesenia described having to be flexible and changing the program depending on the time of year.

Y: So, now in the summer we focus on recreation because it's bright outside, we can go outside and have fields to play and not rely on a school gym permit. Throughout the school year we do different activities that comes from the girls themselves and what they're interested in. They don't feel comfortable engaging in a mixed setting to explore concepts of self-esteem, health, and beauty, having a girl's spa day. They have the chance do it with us though, and I think it's a great program.

\section{Homelessness}

Frank shared the challenges and obstacles faced by one particular newcomer youth he worked with recently. He described her intersecting identities as this:

F: The newcomer, as a matter of fact I saw her today: Muslim, Somali, black, prostitute, homeless, and pregnant.

Frank emphasized that her multiple identities made her life extremely difficult. She came into his organization on her own and he took her in to help her find housing and support. As a devout Muslim himself, the newcomer youth told Frank that she was under the impression he would judge her or make comments about her situation while he tried to help her. This was not the case:

F: So, what I'm saying this individual, they have multiple barriers, but I am going to help her regardless. I have nothing to do with it just because she was Muslim, or she's a prostitute, just because she's going to be bearing a child by wedlock. Those are the things are not for me to judge. The biggest thing that happened is because she's Muslim and because of myself being a Muslim and a religious person, she thought I would be judging her. I have never used that word, "haram" [defined as forbidden in Arabic]. And that made this young girl respect me.

Frank then went to explain that her most imminent need was housing, but the government doesn't provide housing for the homeless. He had to use his own connections and resources to get her help. He also stressed that his relationship with her, or other newcomer youth, should be embedded in trust and professionalism. 
F: That [respect] enabled me to work on her, that took me to the government saying that she is outside, and the government does not give residence to the homeless, because there are so many. I was successful because I said I'm not asking for her, I'm asking for the child that is going to be born and I used my own connections to help. Today is first time she brought the baby here [to the organization], it's one-month old now. She came here, on her own and I got that opportunity of doing something correctly. Because at the end of the day as I say, all these people these newcomer youths who are coming to receive services, they have the right to receive the service and us people who are providing the service have the obligation to give it in a trusting professional way, a truthful way.

\section{Organizational approaches to intersectionality}

The following section outlines participants' grasp of the ways their newcomer youth program works to be more inclusive and welcoming of multiple newcomer youth identities. Key informants provided a wealth of information and examples to highlight their organizations' cognizance of intersectionality, and how it is actively incorporated so that the needs of all newcomer youth are met. Specifically, this last section emphasizes some initiatives taken by organizations; how funding effects their ability to be more inclusive; best practices when working with diverse newcomer youth; and the significance of collaboration and cooperation in the community.

\section{Initiatives}

The two ways key informants shared that their organizations were aware of intersecting identities and made active efforts to address the needs of all newcomer youth, were through antioppressive trainings for staff and a youth survey for clients.

\section{Training and workshops}

Organizations aim to help staff and frontline workers by conducting consistent and comprehensive trainings about discrimination, oppression, and diversity. Participants felt this helped with their understanding of intersectionality because it made them more aware of intersecting identities, diversity, and marginalization. For example, Frank mentioned that the 
training conducted at his organization reminded staff of how diverse the youth are and of

professional boundaries:

F: We have our own training program. For example, we, once a year, we have a whole one week. We do have that. We talk about, diversity training, oppressions... safety, from the service providers, for the youth. We are with the youth, you know what I mean? Very close, we know everything about them so we have to have some boundaries. Some respect. It's good to remind us of uniqueness of every youth we have. How different they are, their experience.

Janice shared that the trainings complemented her theoretical background and made her more cognizant of the different intersecting newcomer youth identities she could potentially interact with.

$\mathrm{J}$ : We always have trainings in anti-oppression and it's for further enhancing the tools for frontline settlement workers, and how to work with newcomers. There's always that piece of anti-oppression in every workshop we always touch base on how to better accommodate our clients. I came from a theoretical background, so a lot of the things that we do in the workshops, I've encountered before, but the fact that they do this workshop, it makes you think of their broader applications. Like they stimulate you to make you think of all the possible ways and youth that you might encounter, and how their experiences are so unique, how their identities work for and against them, how they work together. It reminds you that you should be more diligent about specific practices.

Yesenia talked about the way she thought trainings impact her understanding and ability

to provide services as well. She also mentioned that she noticed there was an increase in

trainings on mental health issues.

Y: Just how can staff be more aware of our language, our body, how can we better and acknowledge our clients. With clients in general and how can we apply intersectionality, work with diversity and experiences that they come from. And I've noticed in the last year or the last few months, we've been giving a lot of training around mental health, inviting facilitators from Cambridge, we got a two-day training on anti-depression and there's another training on professional boundaries. So, I think there's is an effort, and they're investing more dollars into staff training because that's not something our funders even gives. It's great for my own interpretation of what an intersectional approach looks like.

\section{Youth survey}

Yesenia also shared that one way her organization is attempting to analyze their programs and creates new ones is through an online youth survey, in which many of the participants were 
newcomer youth. Specifically, a survey was rolled out to youth in the area which allowed them

to identify concerns and issues they felt affected them the most. Yesenia shared more about the survey:

Y: We worked on a survey and we launched it five or six months ago. We just completed 66 survey inputs from youth around the neighbourhood. It asked about what services are important to you in the neighbourhood, what things are impacting you. The number one in the analysis was that sports and recreation we found was very important for them in the neighbourhood. What they felt most impacted was, they were impacted by...the top three were health, racism, and employment which is interesting, and I would say this in part, a lot of them are newcomers.

She elaborated on the results further:

Y: So, I think as a reflection, that's why I say sports and recreation is big. The other questions were, what issues do you feel most impacted, like "what issues are you most impacted by in the neighbourhood?" And it's a list of all these issues like crime safety, health etc. It's a kind of needs assessment and it was kind of surprising how it was health, racism, and employment.

Speaking more about the 'Health' option on the survey, Yesenia shared how the survey was organized and how it allowed youth to be more specific with their answers. She discussed that youth mostly wrote 'stress', 'anxiety’ and 'dental health' after deciding 'Health' was something that impacted them:

Y: I think there was an option for mental health, like we're talking about stress and anxiety which is mental health. Beside 'health' we had a line to say: specify. We saw more about stress, anxiety, that was their comments... and oh dental as well.

\section{Funding}

When discussing potential ways that these newcomer youth programs could improve or be more inclusive, funding was identified as the biggest restriction for multiple reasons. Key informants cited feeling limited and unheard by government funders, which stifles their ability to apply a comprehensive intersectional lens. The requirement to prioritize serving more recently arrived newcomer youth over older ones, the slow process through which the government listens and acts upon service providers feedback, and limited resources that don't allow for more staff or 
external evaluations are all highlighted below as the ways participants felt limited government funding and oversight hindered their capabilities.

\section{Newer newcomer youth}

Government funded programs have detailed and strict terms that organizations are obligated to follow. One of those includes providing more services to newcomer youth who are newer to the country than others, tracking that data, and reporting it back to the government. This limits the organizations, does not address the needs of all newcomer youth, and creates a hierarchy in service provision. Yesenia described it as such:

Y: So, we do have targets, IRCC has a very strict term on what they identify as newcomers which is like permanent resident, refugees... which we can report on. But like our services are for all, second generation youth who may still have that newcomer experience because of their parents, youth who've been here for years, or those who have become citizens because that doesn't mean anything. They're still experiencing settlement issues.

Janice shared that she thought improving programs wasn't under the organization's

control as much as it was under government funders. She thought the reliance that the

government has on statistics and numbers for meeting their target population (newly arrived

youth) takes away from the potential of the programs:

J: Funding requires many different things that sort of imposes restrictions in a way. For example, we need to have a target for every single youth that we serve. So, that way...that's always been the point. We always need to strive to get that number. So, in terms of certain programs, certain trips, our management would ask us to prioritize you know, new youth over the youth that's been accessing the programs many times, been here longer. I think that's one big barrier, I understand coming from management, they don't want to be unfair, I think it's mainly because the funding requires us to reach these targets, without that we can't have the programs. It's a really big restriction.

She added that she found it difficult to explain and understand the prioritizing of quantity over quality:

J: It pains me to hear youth say, "Why can't we go to this program, just because I'm a citizen?" How do you explain that, how do you answer that question. It's always been hard, the fact that funders value quantity over quality in programs. 
Frank had a different take than the other participants on this situation and felt that newly arrived youth required more support. When the objections that "older" newcomer youth had to their needs not being prioritized came up in conversation, he said:

F: Of course, there is, because the funding is for this [newly arrived youth]. But, it's good because these individuals come, like for example, recently when we are seeing the Syrian refugees, give them the preference, chances first, over those who are here five years, it does make sense to me, it does. I don't see anything.

\section{Bottom-up approach}

Frank was critical of the government's disengagement in other ways, claiming that he doesn't expect them to provoke or influence change. Instead, he thinks that community members are more equipped to make changes and the government should use their insights rather than imposing how an organization's programs should function. Frank stated:

F: Yeah it has to be built from the bottom. Community are the only people that can make a change. Don't expect the federal government to do it. Let's look from the bottom up, rather than them telling you [organizations] what to do. The community should be able to tell you.

He compared it to the way the employees must abide my employer rules, but added that in his organization, newcomer youth voices were still heard and acted upon.

F: Example, whenever, you know I have to do my service according to what my organization is expecting me to do. No different, the government is giving the funding, and agency is funding, do it according to that. But what I have noticed, that this organization, they are providing the service by listening to the youth, like what do you want, rather than the other way around.

When asked whether the government was listening to service providers, he added that some of their requirements would be better informed if they listened to the community and service providers' faster: 
F: Are they listening? They are listening but they're not listening fast enough. That's why we're losing the younger generation into these kind of things that is happening. Whether it be gang violence, whether it be the substance abuse, dropping out of the high school is the perfect example. You know, there is ways that we must turn on to encourage the youth, back in the school. Listen to them, listen to us.

\section{Lacking funding and resources to train and evaluate}

A reason why newcomer youth with intersecting identities might feel unwelcomed was also proposed as a result of low funding. Yesenia said that while her organization was appreciative of volunteers that help during programming, many of them don't have the proper training required when working with newcomer youth or cannot relate to their experiences. She said:

Y: Every program we facilitate we have at least two volunteers. It's great to have volunteers, but you never know, there are a lot of volunteers that are Canadian born who are new to the programs, they come into the program because they want to learn about newcomer youth. They don't know...the awareness and just having that proper training on the language and the support that you use with newcomer youth is important to obtain.

She elaborated by stating that while staff members and service providers are aware and mindful,

they don't always have the time, money, or resources to train volunteers at that same level:

Y: [We are] being very cautious about the language we use because that's what youth can pick up on and making sure that we're not in any way disregarding our youth from their experiences or maybe their ability to express. Like that, training our volunteers is also vital because our program is a community connections program, but it's not always possible.

Moreover, Yesenia also believed that addressing all newcomer youth needs and having a third-

party evaluate the programs for feedback was essential, but required a lot more funding than they

are currently allocated for. She explained it as such:

Y: There are funder limits as to how rigid an organization can work in terms of intersectionality issues because there's only so much of a budget we can work with. How can we tap into all the barriers and address all the issues that our youth are facing and be more inclusive? Maybe it requires more trainings, do we have the budget for that? Maybe it requires more you know, facilitators to come in and have a look at our space and give us feedback on it. I'm sure there's people like that out there, who can give feedback around that, and no we haven't able to do that or explore things like that because it comes down to funding, budgets, and reporting. 


\section{Best practices}

The two ways that key informants interact with newcomer youth is through

programming/workshops or one-on-one formal and informal interactions. Participants discussed

what they thought were best practices in both scenarios when it comes to dealing with diverse youth with multiple identities.

\section{Communication}

Key informants expressed what they thought were the best practices to use when reaching out and dealing with newcomer youth, especially those who are new and might feel displaced or discounted. Janice described her personal approach when she greets them:

J: I always ask youth, how are you? It's as simple as that. Whenever I call them, whenever I see them, I just ask, how are you? Then I look into their eyes and they say, "I'm ok" and if I think they're not ok, I ask do you want to talk? It's as simple as do you want to talk or you can always talk to me? And I more adamant on follow ups too, whether they are attending programs or not, I'm always checking and following up with them.

Frank detailed some of his best practices and even attributes representation as a factor that dictate interactions with newcomer youth. He explains it as such:

F: Make it easier for them to open up. Be genuine, don't give them the false hope. Respect them, love them, genuinely, and support them.

He added that he thinks when staff are representative of the population they are serving, not only are the youth more comfortable, but the frontline workers are in a better position to feel and provide empathy because they can relate:

F: The staff itself is more you know, different orientation, different ethnic backgrounds, we're very diverse. It's representation that matters. The services that we are providing and being accepting, the words that I would be using is empathy, rather than sympathy. Ok, so staff who are relatable they are in the better position to offering empathy. Newcomers can interact and feel more included and welcome.

Janice and Yesenia also outlined how critical communication through cellphones and social media is for newcomer youth. Often youth will have imminent needs and questions, and 
since they all have cellphones and engage on social media, organizations took the initiative to

use them as a tool for communicating.

J: We don't only have cellphones; we also have a Facebook page. Youth don't use email, they're in high school. Our [work] emails have no use, except for partnership and communication with the team, but in terms of youth, it's important for you to have cell phones at least a clear way to communicate with them so they know to reach us. Even in terms of one on ones, check ins, follow ups, work cells are amazing, whichever youth I interact with I always give them my cell phone number just because you never know.

Yesenia shared that these methods of communication are not only easy and meaningful, but also

useful to track how often and to what extent newcomer youth are interacting with them.

Y: In most cases, that's our way of measuring, so they're actually coming again, or they added us on Facebook and they're messaging us, they're interacting. Youth are constantly contacting my cell phone because my contact is on the general flyer or they need support right away. We're really active on our social media and they're Facebook messaging all the time about support and things they need all the time.

\section{Civic engagement and recognition}

While Janice was clear that sports and recreation are essential programs to have, her idea

of best practices and programs entails more civic engagement and more community involvement.

She believed that civic engagement leads to a more open mind, and that getting involved is

essential to feeing like you belong, especially for those who might feel disregarded or

marginalized because of their identities. She described it like this:

J: So, I think, for me, it's my opinion on how I want to see it. I don't want to see a lot of sports programs happening, there' already a lot of them, which is amazing, I definitely value sports engagements, how it plays a role in youth mental health and physical health. It's like I told you, there's already a lot of that, we need more diversity in terms of programming. More skill based programs, more civic engagements, that's one way for youth to be more accustomed to their community, to open their minds, feel a sense of belonging, get involved. Especially when you have so many conflicting and discounted identities, it can help.

Being recognized for community involvement and civic engagement is also cited as a

positive motivator for newcomer youth. Janice explained that their programs offer youth to get 
involved and have in return received recognition from community members and other

organizations.

$\mathrm{J}$ : With our programs, we give them an opportunity to do outings in every cohort, it opens their mind to many different aspects to their community, engaging them with people from many different backgrounds. They've received awards and been invited to events, a couple of them recently cut the opening ribbon for an event, and you can see they're very happy with themselves, even their parents are.

Yesenia shared that when newcomer youth volunteered with one corporate organization

in the community, it resulted in the corporate group supporting their program and fundraising for

them. The benefits of community involvement are outlined by her below:

Y: It was at one charity event, that team [corporate organization] is made up of a lot of retired teachers, professionals, so they were like "wow newcomer youth, you guys do so much work, let us give you some pocket of funding" They give mentorship support, staff support, they're willing to jump and give that support, because they recognize the impact and effort the organization and newcomer youth are making.

\section{Collaborative efforts}

Cooperation and collaborative measures between organizations are discussed to show that they can also positively impact newcomer youth experiences when accessing services and programs. Frank discusses collaborating with Toronto Police Service (TPS) to settle a dispute amongst local mosques. He claims that two Somali mosques in the same area, who had differing opinions, refused to interact or let their kids get involved in the community in fear that they would mingle with the opposing party. He explained it like this:

F: In this organization, I have witness[ed] that they have, they were the first organization to bring the Somalians, they took the lead from the Somalian organizations to be reunited and that's a mediation. The mediation of those mosques...there was a dispute, there was a resolution with this organization with the help of [local] division two years ago.

When talking about how mediation changed the clientele of the newcomer population, he said it increased dramatically since the issue was resolved and there was no restriction on the newcomer youth: 
F: Because of that, it [population] has increased I would say more than $50 \%$. That group of people because they're predominantly they're here, they're you know, big organization, they're very close by. Their feelings and business effects our newcomer kids. Muslim youth who are deprived from the services because they weren't allowed to get involved, they're the ones who needed it and are receiving the most services now. We started it here, with the TPS, the Toronto Police Service, together.

Yesenia claimed that while other programs in their neighborhood were more concerned with settlement steps and were process based, her organization is more about programming, activities, and social/recreational initiatives. She described that was an obvious need in other neighbourhood for these types of services, which is why they started to reach out and expand their services to other places. This allowed them to meet the needs of newcomer youth in areas where more diverse and interactive services were scarce.

Y: There's been a decline in newcomer youth settling in this neighbourhood and there's been an increase in newcomers in north west from here. So, the west... up north from here and we've been bringing our programs there to meet the needs of newcomer youth. Because there's no youth programs, there's always settlement services programs, and there's youth programs, but there's no newcomer youth programs. There's a clear difference between those two programs, they're not the same as a newcomer youth program.

She added that by being able to collaborate and work with other agencies in different areas, they

can provide comprehensive services and address all needs of newcomer youth

Y: Other places there are more workshop and process based. We're more about programming and activities, and how to include youth. Because our definition is how we can include them, match them with volunteers, and get them more engaged in the community. So, I think we've been able to fill in the gaps by partnering with other agencies who serve newcomer youth and start programs hand in hand in those new places. It doesn't make sense to saturate services, when the population is so diverse and has intersecting needs and identities. 


\section{$\underline{\text { Discussion }}$}

The aim of this study was to grasp the comprehension and awareness that newcomer youth programs in Toronto have of intersectionality and its potential applications. Newcomer youth are part of a diverse group of migrants who affiliate with multiple identity groups based on, race, religion, gender, sexuality, socio-economic status etc. An intersectional approach considers the existence of these multiple identities, and therefore recognizes how multiple inequalities, inclusions, and exclusions function at the same time. An intersectional understanding and lens is one that incorporates the main idea proposed by intersectionality; acknowledging that our identities overlap and intersect to influence our experiences with oppression and marginalization.

By speaking with three key informants, who are service providers in newcomer youth programs in Toronto, this study tried to gauge programs and services for newcomer youth that are aware of this reality and use it as a tool to include youth who might be excluded and discounted because of their multiple intersecting identities. In the findings, key informants highlight experiences they had with newcomer youth, practices, and initiatives, both personal and organizational, that are informed through an intersectional lens and worked towards addressing the needs of newcomer youth with multiple identities.

The section below discusses and sheds light on some of the main themes and ideas proposed by key informants. To fully comprehend key informants understanding of intersectionality it was first imperative to let them explain how and why they thought there was a need for intersectionality. This study allowed key informants to share the ways they thought having intersecting identities shaped the overall settlement experiences of newcomer youth and how it proved to be a barrier when accessing services or trying to integrate. Their insights are 
elaborated and supported by the concepts and findings from the literature review in this paper. Moreover, key findings that were not mentioned or referred to in the literature review are also present in the discussions below. For example, details about the experiences of newcomer Muslim girls, the tensions in youth spaces, and the significance of community involvement programs are mentioned below as they prove to be crucial and worthwhile points, despite being unprecedented findings in the literature review section.

Exploring, discussing, and dissecting the incorporation of an intersectional lens is divided into two primary categories: i) how service providers understand and interpret intersectionality when providing services; and ii) how organizations curate programs and initiatives that are informed by intersectionality and work towards the inclusiveness of all newcomer youth. While these categories are not mutually exclusive, as one can and often does inform the other, for this research study they are discussed separately to highlight specific instances and maintain clarity.

\section{$\underline{\text { Service providers }}$}

This section outlines insights that key informants provided regarding newcomer youth with multiple identities and intersectionality. By analyzing key informants' definition, need, and application of intersectionality, it is easy to draw similarities, as well as new insights, between their observations and existing literature. Moreover, it is important to mention key informants' discussions about barriers that newcomer youth with multiple identities face because it influences their understanding and application of intersectionality. Their interpretation, usefulness, and need for intersectionality reflects and stems from their direct experience with newcomer youth. 


\section{Defining intersectionality}

Despite using different terminology and phrasing to define intersectionality, key informants expressed similar views and generally perceived intersectionality as recognition that peoples different identities shape their access to services and their experiences. For example, Janice claimed that accommodation is engrained in newcomer youth programs because of the different struggles that multiple identities carry, Frank thought it was more about the barriers that people with intersecting identities have, and the need to practice acceptance in organizations, and Yesenia believed that intersectionality was the mixing of identities that shape how individuals see the world and that it "determines their paths and what kind of programs they want to access...if they feel included". The takeaway from these interpretations of what intersectionality entails is that all participants believe there is onus on organizations and programs to acknowledge and be inclusive of intersecting identities. Each key informant mentioned something that connected intersectionality and intersecting identities as factors that determine the accessibility of newcomer youth into programs and services.

\section{Need for and applying intersectionality}

Participants provided a wealth of information, stories, and experiences that emphasized the tribulations that newcomer youth with intersecting identities must endure before and during participation in programs, and ultimately shared why intersectionality should be adopted. For example, low socio-economic status was attributed to many of the limitations that newcomer youth faced. Financial responsibilities and stress from being a newcomer require youth to work multiple jobs, and were mentioned by Yesenia and Frank to exemplify how these factors influence their interaction with the programs. Yesenia shared that some youth don't have time for programs because of multiple jobs, or because their parents are working multiple jobs and 
they must take up responsibilities at home. Furthermore, the youth survey conducted by Yesenia's organization also indicated that youth thought employment was one of the issues they felt impacted them the most. This suggests that money and jobs, whether for financial independence or to support their families, is something that concerns newcomer youth. Frank noticed the same situation and added that financial stress is something many newcomer youth come to programs to relieve when they have the time. Similarly, Janice discussed that some newcomer youth don't have the resources, such as TTC tokens, to attend programs around the city which is why her organization always offers them. These observations are supported by Francis and Yan's (2016) study about gaps and bridges for African newcomer youth in Vancouver. Their study showed that heavy financial and familial responsibilities can be a determining factor that often results in uneven attendance and participation of newcomer youth in programs (p. 90).

Barriers surrounding youth who identify as LGBTQ and those suffering from mental health issues were also alluded to in the findings and literature review of this study. The one commonality between the two was that newcomer youth disclose these identities to service providers. Mental health was one of the other options that youth expressed they felt impacted them the most in Yesenia's organizations' survey. The incident that Janice shared with the youth who was diagnosed with schizophrenia exemplifies the hidden and personal nature of living with mental illnesses. Janice's concern was that there was no way for her to better support the youth without knowing the situation, which is why she now always sincerely asks how youth are doing and follows up with them diligently, regardless of whether they are attending programs. Moreover, Yesenia and Janice expressed that they would like to be more inclusive and inviting of LGBTQ identities but don't want to pressure anyone to share or disclose these matters. 
Interestingly, research alludes to this also being a result of intersecting identities. Daley et al. (2008) indicate that newcomer youth have trouble "coming out" because of their combined experiences dealing with racism, classism, and homophobia (p. 21). In fact, one of the youth workers in their study called it a process of one insecurity heightening another insecurity (Daley et al., 2008, p. 22). Yesenia shared that she has attempted to make an active effort to invite and be inclusive of LGBTQ identities, such as planning a trip to the Pride Parade and trying to incorporate more LGBTQ posters in their youth space, but finds it difficult for varying reasons. A positive to take away from this is that key informants are aware they are not necessarily meeting the needs of LGBTQ youth, because as Travers et. al (2010) discuss, it is much more dangerous when organizations are in pervasive denial that LGBTQ youth exist and/or never provide any room for that dialogue to occur (p. 195). While they might be limited because of the lack of "out" youth, key informants are making efforts towards trying to open the discussion and be welcoming when youth are ready to disclose.

A major finding about how multiple identities can intersect, and one that warrants a discussion because of its consistent reoccurrence during participant interviews, is the barriers faced by Muslim girls in newcomer youth programs. The need for an intersectional lens that considers ways that Muslim girls are excluded and limited from programming was alluded to by key informants. Yesenia and Janice both shared that girls only programs were scarce in their neighborhoods, and Frank stated that girls' attendance was low because of programs that mixed boys and girls together in his organization. However, Yesenia's and Frank's approaches to mediate some of these barriers were different. Yesenia explained that her organization went through various obstacles to curate programs specific to the needs of girls, both Muslim and nonMuslim, who needed a safe and comfortable space to participate. Frank, however, stated that he 
thought some of the low Muslim girl attendance could be attributed to parents being afraid and unsure as to what their kids do and learn in programs, and thus open communication and transparency between parents and service providers was essential. Another distinction to note is that Yesenia stated newcomer Muslim girls themselves expressed disinterest in mixed programming, whereas Frank believed it was parents and religious leaders making the final decision. Yesenia mentioned that Muslim girls choose themselves not to want to play sports with boys, and likewise, studies have given Muslim girls that same agency. Strandbu (2005) exemplified this when talking about a participant in her study who explained why it was unpleasant for her to exercise with males. The participant cited her personal interpretation of religion and culture as her reasoning and stated clearly that her parents were not very strict in this matter (Strandbu, 2005, p. 32). Similarly, Frank's insight about parents and religious leaders being uncertain about what some newcomer youth programs entail is also supported by Francis and Yan (2016). Their study states that parents are often weary of sending their kids to newcomer youth programs in fear that children might pick up undesirable behaviors from facilitators, or other youth, and forget their roots (Francis \& Yan, 2016, p. 90).

Participants being able to identify that certain identities and groups face heightened barriers when accessing programs, while also realizing that steps must be taken to help minimize them, is what working through an intersectional lens means. Their ability to provide clear examples of ways they have seen certain identities be excluded from programs highlights that they are aware of the need and significance of intersectionality. When talking about how intersectionality affects key informants' personal approaches to providing support, they mentioned their backgrounds influencing their application of intersectionality. Yesenia said that she comes from very similar experiences as newcomer youth, which makes her more passionate 
to understand intersectionality and apply it to her services. Janice was once a newcomer youth herself, and stated that her theoretical understanding of intersectionality from school provides her with the right tools that are then enhanced by frontline and interactive work involving newcomer youth. Frank shared that his experience in court proceedings made him more aware of how intersecting identities and intersectionality work, and made him more careful when dealing with youth with more than one marginalized identity. These observations from key informants cannot be understated because their influence and frontline work not only gives perspective into their interactions with newcomer youth, but is also in part a reflection of their organizations work. By emphasizing their cognizance of intersectionality, they are better equipped to advocate for change and improvements that meet the needs of newcomer youth with multiple intersecting identities.

\section{Newcomer youth organizations and programs}

This following section brings forth the ways that newcomer youth organizations work with an intersectional approach and towards being more inclusive. Key informants drew upon initiatives and efforts that their organizations were a part of, both within the organization and in the community, to exemplify their cognizance and comprehension of intersectionality. Participants were also not hesitant to point out what they thought was limiting their organization from improving, namely lack of and restrictions from government funding.

\section{Initiatives within organizations}

Given the diverse and unique staff and frontline workers at newcomer youth organizations, everyone's understanding of oppression, discrimination, and ultimately, intersectionality is not the same. Therefore, it is imperative that organizations take a proactive stance on training and filling in knowledge gaps for those staff members who may be 
uninformed of certain oppressions or intersectional approaches. Participants praised the antioppression training sessions that were regularly conducted in their workplaces, for many reasons. The trainings not only allowed them to learn from knowledgeable facilitators and from one another, but Janice thought they also serve as a reminder to be more attentive to specific practices. Yi et al (2016) discuss how lack of knowledge training and difficulty assessing clients were barriers that service providers cite as hindering the quality and level of service they can provide to newcomer youth (p. 1082-83), so workshops like these are essential to have a wellrounded understanding of factors that affect any given population. Unfortunately, the duration and consistency of such trainings can be unpredictable as they are solely dependent on money and resources that the newcomer agency has available. Yesenia shared that the training and workshops her organization conducts are not part of the government provided funding; thus, it is often the agencies investing their time and resources because they realize the impact and importance of training.

Currant et al. (2010) mention the discrepancy between what the government expects newcomer youth organizations to be able to do and the reality of the funding they provide. The report states that government funders expect organizations to do research and tackle complex social issues, but organizations are unable and underfunded to hire staff with the proper training and education to do so because the wages are so low (p. 24). In fact, sometimes wages can be so minimal and insufficient to hire new staff, that service providers rely on the help of volunteers to facilitate and support their initiatives and programs (Van der Meulen et al., 2010), something Yesenia shared as well. Yesenia added that many volunteers do not have the same mindfulness or the comprehension that staff have of newcomer youth identities and issues, yet they facilitate programs and interact in similar ways as service providers would with newcomer youth. In 
example, while youth spaces are generally considered to be welcoming, participants elaborated on some of the tensions and animosity that can arise between newcomer youth when they are sharing the space. Workers need to be able to think critically and quickly in these instances and have the capability to navigate difficult conversations, something volunteers might not be able to do.

Another informative insight that key informants shared pertained to the government requirement and expectation to serve newer newcomer youth over those who have been here longer. The CAMH (2014) report on homeless newcomer youth indicated that some youth fear there is prioritization in the service system to serve more recently arrived youth (p. 27), and the findings in this study confirm this notion. Newcomer youth should not be turned away or penalized based on the length of their time in Canada because they might still be facing some of the same settlement issues and concerns. However, organizations are obligated to report to funders on the clientele the funding serves and therefore give preference to more recently arrived youth.

The aforementioned youth survey and the girls only programs are two other ways that organizations make active efforts to be more inclusive and recognize that intersectionality is a useful tool. While the youth survey yielded interesting results that highlight the diverse and intersecting identities that newcomer youth have, it is unclear how the results are going to be translated into action. However, the idea to conduct a survey that was detail orientated and allowed room for youth to be as specific as possible ensures that their voices are present in service provision. Actions like these are also discussed by Frank who said that despite the government providing guidelines and imposing restrictions on organizations it funds, his workplace still values the voices and experiences of newcomer youth. The discrepancies between 
what the government might understand and what frontline workers know is what often becomes a bone of contention; participants claimed that the government was not listening or was not providing support fast enough to address the needs of marginalized and excluded newcomer youth. The second initiative taken by Yesenia's organization, which exemplifies their commitment to serving the intersecting identities of newcomer youth, is the girls' programs created to include Muslim newcomer girls. Not only are they filling in a crucial necessity in the area and youth population, but also their determination to overcome various obstacles they faced when trying to keep the program running, shows the value they put on newcomer youth who are marginalized. An intersectionality driven approach requires organizations to be flexible, innovative, and proactive when creating programs for those who have multiple intersecting identities. If organizations can commit to continuing to make their programs more inclusive and recognizing the barriers that intersectionality alludes to, they can influence the way newcomer youth with intersecting identities engage and seek support.

\section{Initiatives within the community}

Newcomer youth organizations do not exist in a vacuum; nor do they have one consistent and fixed clientele. Newcomer youth are constantly moving in and out of the programs for multiple reasons. Organizations need to maintain a connection with the community and be aware of the issues that are persisting around them, to ensure that their programs are not shortsighted or irrelevant for newcomer youth needs. Key informants expressed a similar sentiment in their discussion about ways their organizations were involved in the community and considered how the needs of youth with intersecting identities were influenced by their surroundings. For example, Frank's story about his organization being the facilitator, along with police officers and 
religious leaders, to settle a dispute between two mosques in their area, indicates the lengths organizations will go to and the attention they give to their surroundings. The newcomer youth at the mosques were being told not to attend the newcomer youth programs at Frank's organization, both to boycott other local organizations and in order for one group to avoid having to interact with the other. By taking the initiative to mediate the discussion mainly because newcomer youth were being deprived of services and support, his organization shows their cognizance of how crucial it is to understand the influence of intersecting identities in newcomer youths' lives.

Developing strong ties and partnerships with other newcomer youth agencies is also crucial. Van Ngo (2009) claims that supporting newcomer youth to deal with the diverse and complex issues they have requires a substantial amount of cooperation and collaboration between programs (p. 94). Similarly, Yi et al. (2016) highlight that an integrated approach which stimulates connections with programs that address different issues and inequalities is vital for newcomer youth with intersecting identities. This idea is consistent with Yesenia's reasoning behind her program expanding services to other neighborhoods. Because there existed numerous typical settlement workshops and programs, but there were no creative or interactive programs north west of Toronto, her organization started to outreach and provide services in new areas. She also mentioned that there were no specific newcomer youth programs in certain areas, that there were newcomer programs and youth programs, but those are not the same as newcomer youth programs and are neither efficient or sufficient in addressing newcomer youth needs. Not only are newcomer youth new, but they are also young, and the ways that just those two identities intersect requires nuanced and tailored services that separate programs are not capable of addressing. Being able to recognize this reality and by staying updated on the service 
provisions of certain areas, organizations make conscious and sound efforts that place newcomer youth intersecting identity needs at the forefront.

Lastly, encouraging newcomer youth involvement in the community is also a good example of how intersectionality is used to make programs more effective. Intersectionality suggests that those with multiple intersecting identities face barriers and increased marginalization, and when coupled with ideas from social exclusion and inclusion theory, it is implied that feelings of exclusion resulting from marginalized identities can create a lack of belonging in society and communities. Newcomer youth are part of the group of individuals whose multiple identities define and determine their access to programs and services, and dictate many of their feelings, or lack of therein, about belonging and inclusion in society. Janice's observation that civic engagement and community involvement should be emphasized just as much as sport and recreation for newcomer youth is grounded in those same ideas. Not only does it make them feel like participating and contributing members in society, but as Janice shared, many newcomer youth get recognized for their involvement in the community and are rewarded for it. Her organization is making an active effort to support these initiatives because they've seen the positive impact it has on newcomer youth who otherwise feel discounted or unwelcome because of their social positions. So, while some of their identities label them as marginalized and make it difficult to feel social inclusion, by participating in community involvement through the gateway that Janice's program facilitates, they can overcome that barrier. Literature suggests that community involvement is a commitment that can present opportunities for youth to be recognized as valuable members of society (Curran et al., 2010, iii). Curran et al. (2010) also mention that newcomer youth agencies are in a good position to be mediators and support youth 
to make this connection, as these strength and bonds in the community help towards meeting their needs better (p. iii). 


\section{$\underline{\text { Recommendations }}$}

Newcomer youth arrive in Canada with intersecting identities and various needs. Community organizations are often at the forefront, along with schools, to provide support and services to youth. The findings of this study explore how intersectionality is understood and applied by service providers and newcomer youth organizations in Toronto. The practical application of intersectionality, as it pertains to newcomer youth programs, is to first recognize how intersecting identities work to create more barriers and accessibility issues for newcomer youth, and then actively work to help minimize those barriers in their programs.

Overall, the results show that while service providers are aware of what intersectionality means and can easily justify its use by sharing how intersecting identities work to marginalize youth, there are certain setbacks and limitations keeping them from being more inclusive and proactive about these issues. The findings in this study are useful for key informants and organizations who can be reflexive and map out their strengths and weaknesses. And the organizations can learn from one another's practices, for example, youth surveys and the type of programming described by some key informants.

This study is also significant to the body of research on newcomer youth in Toronto, because the study explores different ways their multiple identities work and how organizations are, or can, cater to their needs better. The discussions below emphasize some recommendations and suggestions to enhance the application of intersectionality in newcomer youth organizations.

Newcomer youth are not a homogenous group and thus their needs should also not be homogenized. As participants showed through the experiences they shared about newcomer youth with intersecting identities, it is not effective to generalize their needs. Key informants expressed being unaware of the complexities and hardships that some newcomer youth endure 
because they relied on newcomer youth to disclose these factors to them. Individual family dynamics, mental health issues, and coming to terms with one's identity are personal and private processes that service providers may not have full access to. Yet all these nuances intersect and dictate newcomer youths' participation, involvement, and interactions with programs. In fact, some of the issues they endure could be resolved through the resources and expertise that service providers and organizations have; therefore, it is unfortunate that their difficulties and challenges go amiss by service providers.

The recommendation here is to create more safe spaces and have open conversations about some of the more pressing concerns newcomer youth have, as indicated by the key informants. Informal trips or conversation circles on specific topics, such as mental health issues, financial stress, employment, discrimination etc. might stimulate a natural and healthy conversation. Even if newcomer youth are not comfortable sharing in front of others, they'll at least be aware that, when and if they are ready to share, the right support and resources are present in the organizations. Having a safe space or someone to organize an informed conversation circle requires expertise and resources, but the potential for positive results and payoff is very high. There needs to be a clear indication in newcomer youth organizations that intersecting identities and issues are understood so that newcomer youth are less intimated to open up and share.

Secondly, key informants alluded to the criteria and restrictions imposed by government funding as a major barrier to applying an intersectional approach. The government's focus on numbers and outputs rather than the value of outcomes, is cited as a reason that newcomer youth programs are not as inclusive and successful as they should be. It would be beneficial if the 
government's focus were on the real-life results and development of newcomer youth, rather than just the numbers attending the programs.

For the same recordkeeping/accountability reasons, organizations have had to turn away youth who have been in Canada longer and are still experiencing settlement and integration issues because the funding and proposals clearly prioritize newer newcomer youth. Programs are required to report back to the government about how the money was used, which must coincide with the initial funding proposals or else they get defunded. If there is a high number of "older" newcomer youth who still seek services and whose needs are not being met, it presumably indicates that services have not been adequate at recognizing and applying a comprehensive longstanding approach to programs. Perhaps instead, the government should consider creating funding and criteria that take a long-term focused approach rather than just time spent in Canada as being an indicator of settlement. They should incorporate a more robust long-term funding plan that considers the long-term goals, support, and settlement needs for newcomer youth. If from the start, funders consider the future needs and goals of the newly arrived youth and actively work towards meeting them, the numbers seeking significant support after several years here would be much lower since their needs were identified and included earlier in the process.

Lastly, diversifying programs to reflect different kinds of needs that newcomer youth have is also suggested. Toronto hosts a plethora of sports and recreational programs for youth, many of which are free and accessible to newcomer youth. The positive impact of community sports on newcomer youth is studied and exemplified by researchers, however, new and more innovative programs are also needed. Programs that encourage community involvement and volunteering can enhance newcomer youth communication, self-confidence, and leadership skills immensely. As discussed throughout this paper, an intersectional lens recognizes that certain 
identities combine as multiple forms of oppression that may make newcomer youth feel unwelcome in society. To counter this, organizations should adopt more programs that aim to make them feel connected and included in society and the community. Steering away from standard programs to develop something that helps newcomer youth sense of belonging and makes them feel active participants in society are needed and encouraged. 


\section{Conclusion}

The goal of this study was to explore service providers' comprehension of intersectionality, their interactions with newcomer youth who have intersecting identities, and the existing intersectionality driven frameworks that newcomer youth organizations have in place. Community organizations and agencies provide various types of support and services to newcomer youth and the findings in this study show that newcomer youth organizations and service providers make an active effort to be inclusive of newcomer youth who have multiple identities. Findings also highlighted their cognizance of how and in what ways intersectionality helps them provide more comprehensive services. Specifically, three in-depth audio interviews of key informants, who are service provider's in newcomer youth programs in Toronto, were conducted to study how participants defined intersectionality, how they thought having multiple intersecting identities affect newcomer youth settlement experiences, and lastly how ideas and premises that intersectionality proposes informed their own, and the organizations, service provisions. Results emphasize some positive and successful initiatives that organizations have taken through an intersectional lens, as well as highlighting the factors that limited them.

Benefits of this research are embedded in the idea that the presence of intersectionality driven services for newcomer youth not only distinctly impact their resettlement, but can also be a meaningful tool and resource to ensure that services are inclusive and accessible to all newcomer youth. If services, support, and initiatives are not tailored to the specific needs of all newcomer youth identities, they are not entirely accessible. Service providers and organizations can learn more about applying an intersectional framework to programs, as well as see potential challenges that might arise through some of the discussions in this paper. 
However, a limitation of this research is that it is not generalizable to all newcomer youth, service providers, or newcomer youth programs in Toronto. Time constraints and recruitment challenges made it difficult to get a larger sample size for key informants. Additionally, for future research it would be beneficial to expand the scope of newcomer youth organizations to beyond Toronto and look at the Greater Toronto Area instead. Furthermore, future research could also incorporate the voices of newcomer youth themselves to see whether they thought the needs arising from their intersectional identities were being met or not, as well as the voices of other staff members in organizations instead of just service providers. In addition, future research could also consider comparing more precisely how intersecting identities affect male vs. female, and different immigration status (immigrant, refugee, undocumented etc.) newcomer youth and their needs differently. 


\section{Appendix A: Ryerson Ethics Board Approval Letter}

REB 2017-179

Project Title: Examining the Application of Intersectionality in Programs and Services for Newcomer Youth in Toronto

\section{Dear Fatima Saher,}

The Research Ethics Board has completed the review of your submission. Your research project is now approved for a one year period as of Jun 16, 2017. The approval letter is attached in Adobe Acrobat (PDF) format.

Congratulations and best of luck with the project.

Please note that this approval is for one year only and will expire on June 16, 2018. Shortly before the expiry date a request to complete an annual report will be automatically sent to you. Completion of the annual report takes only a few minutes, enables the collection of information required by federal guidelines and when processed will allow the protocol to remain active for another year.

Please note that REB approval policies require that you adhere strictly to the protocol as last reviewed by the REB. Any modifications must be approved by the Board before they are implemented. Adverse or unexpected events must be reported to the REB as soon as possible with an indication from the Principal Investigator as to how, in the view of the Principal Investigator, these events affect the continuation of the protocol.

Please quote your REB file number (REB 2017-179) on future correspondence.

If you have any questions regarding your submission or the review process, please do not hesitate to get in touch with the Research Ethics Board (contact information below).

No research involving humans shall begin without the prior approval of the Research Ethics Board.

This is part of the permanent record respecting or associated with a research ethics application submitted to Ryerson University.

NOTE: This email account (rebchair@ryerson.ca) is monitored by multiple individuals. If you wish to contact a specific member of the Research Ethics Board, please do so directly.

Yours sincerely,

Zakiya Atcha, MSW

Research Ethics Co-Ordinator 


\title{
Appendix B: Recruitment Poster
}

RYERSON

UNIVERSITY

\section{PARTICIPANTS NEEDED FOR RESEARCH ON NEWCOMER YOUTH SERVICES}

\author{
Are You:
}

- A current social worker or service provider at a newcomer youth program or agency - $\quad$ Someone who has experience dealing one-on one with newcomer youth with diverse backgrounds for at least one year

- $\quad$ Someone with knowledge and understanding of the programs and services your workplace offers to newcomer youth

If you answered yes to the above noted questions you are invited to volunteer in this study on newcomer youth services and programs in Toronto

You will be asked to schedule a meeting to discuss and answer a series of questions about your experience with newcomer youth who belong to or identify with multiple identity groups (i.e., race, gender, socio-economic status, sexuality etc.) You will be asked about your understanding of how the programs and service in your workplace work through an intersectional lens. Participation in this study will take approximately 80 minutes of participants' time.

This research is being done as partial fulfillment for a Master's degree in Immigration and Settlement Studies and is being supervised by Dr. Kiaras Gharabaghi: k.gharabaghi@ryerson.ca

If you are interested in participating in this study or for more information, please contact: Fatima Saher, Masters of Arts (MA) candidate in Immigration and Settlement Studies: fsaher@ryerson.ca:

This research study has been reviewed and approved by the Ryerson University Research Ethics Board REB Protocol (2017-179) 


\section{Appendix C: Recruitment Script (email and phone)}

\section{Email, phone or in-person recruitment script template}

$\mathrm{Hi}$

My name is Fatima Saher, a Masters of Arts (MA) candidate in the Immigration and Settlement program at Ryerson University. This research is being done as part of my Major Research Paper and my supervisor's name is Kiaras Gharabaghi (k.gharabaghi@ryerson.ca)

I am conducting research on newcomer youth services in Toronto, specifically on how intersectionality is applied in programs to ensure all newcomer youth are welcomed and adhered to. I am contacting you to see if you, or someone else in your workplace, might be interested in participating in this research study.

To participate, you need to be a current social worker/service provider in a settlement or community agency that provides services and caters to newcomer youth needs. If you agree to volunteer, you will be asked to schedule a meeting with me at a mutually convenient time and place to discuss and answer a series of questions about your experience with newcomer youth who have multiple intersecting identities (i.e., race, gender, socio-economic status, sexuality etc.) and your understanding of how the programs and service in your workplace work through an intersectional lens. Anonymity and confidentiality will be obtained through out the whole research process. Participation in this study will take approximately 80 minutes of participants' time.

I would like to assure you that the study has been reviewed and received ethics clearing through Ryerson Ethics Board (2017-179). Participation is completely voluntary. The interview will be tape recorded to facilitate analysis, although you can request the tape to be turned off at any time.

I write to also ask for your assistance in sharing this information with colleagues in your workplace who fit the demographic and interested participants should please contact me at fsaher@ryerson.ca. I will respond with an email to provide more information.

Thank you for your time and assistance.

Sincerely,

Fatima Saher 


\section{Appendix D: Interview Guide}

\section{Background:}

1. What is your current work title at your place of employment?

2. How long have you been in this position?

3. What are your primary responsibilities at this position?

Could you describe a typical day for you at work?

How often and to what extent do you interact with newcomer youth?

4. How do you think having multiple identities shape the resettlement experience for newcomer youth?

5. How do you think it affects their access to services, programs, and support?

6. If you can do so, how would you define the term intersectionality?

7. What role do you think intersectionality plays in the resettlement of newcomer youth?

How does it shape their experience in Toronto?

8. How do you think an intersectional lens would help an organization be more inclusive?

9. Do you think your, or the organizations, understanding of intersectionality is well reflected in the services?

10. How does your understanding of intersecting identities shape and enhance your ability to provide services to newcomer youth?

\section{Questions about the organization:}

11. How are newcomer youth referred to your agency?

12. In what ways is the population of this clientele diverse?

13. Can you outline some of the main programs and services that are offered to newcomer youth?

a. Which program/service do newcomer youth access the most?

b. What is the longest running and the newest program/service to be incorporated?

c. Which one, if any, do you think is most accessible for newcomer youth with multiple identities?

14. Are there any groups who might face barriers accessing one of your programs?

a. What has or can be done to resolve this?

b. Do you think using an intersectional framework to address this need would be beneficial? Why or why not?

15. What, in your experience, are the main struggles for newcomer youth with multiple identities?

a. In your opinion, do you think this organization is doing a good job at combating such struggles?

16. Could you highlight some other ways you think your organization works to be more inclusive of newcomer youth who have various identities? 
17. How do you think the programs/services have changed in the past 5 years to reflect the needs of the newcomer youth population?

18. Where and how would you like to see these programs and services in 5 years?

\section{Intersectionality related questions:}

19. Have any workshops/meetings/trainings at your workplace explicitly addressed the role and application of intersectionality when dealing with newcomer youth?

a. If yes, was it helpful?

b. If not, do you think it would be worthwhile and useful to have one?

20. How has your understanding of multiple identities/intersectionality changed or evolved since you started working here?

21. What factors do you take into consideration when dealing specifically with newcomer youth with intersectional identities?

22. What do you think are the best practices that make for a successful interaction with the newcomer youth population?

23. Are there any specific intersections of newcomer youth identities that are more common in your organization than others? (Ex: racialized queer newcomer youth or transgender disabled newcomer youth?

24. Could you describe a specific incident you can think of where you were working with a newcomer youth with multiple intersecting identities?

a. How did that change the way you could support and provide them service?

b. Was there anything you would do differently?

c. Do you think you could provide sufficient support/resources in this interaction? 


\section{$\underline{\text { References }}$}

Adascalitei, A.-M. (2016). Between Theory and Practice in the Fight Against Social Exclusion. Research and Science Today; Targu-Jiu, 10-16.

Agyekum, B., \& Newbold, K. B. (2016). Sense of Place and Mental Wellness of Visible Minority Immigrants in Hamilton, Ontario: Revelations from Key Informants. Canadian Ethnic Studies; Calgary, 48(1), 101-122.

Aylward, C. (2011). Intersectionality: Crossing the Theoretical and Praxis Divide. Journal of Critical Race Inquiry, 1(1). Retrieved from http://ojs.library.queensu.ca/index.php/CRI/article/view/3549

Bauder, H. (2003). Cultural representations of immigrant workers by service providers and employers. Journal of International Migration and Integration / Revue de L'integration et de La Migration Internationale, 4(3), 415-438. https://doi.org/10.1007/s12134-003-1028-9

Berman, H., Mulcahy, G. A., Forchuk, C., Edmunds, K. A., Haldenby, A., \& Lopez, R. (2009). Uprooted and Displaced: A Critical Narrative Study of Homeless, Aboriginal, and Newcomer Girls in Canada. Issues in Mental Health Nursing, 30(7), 418-430.

https://doi.org/10.1080/01612840802624475

Brotman, S., \& Lee, E. O. J. (2011). Exploring Gender and Sexuality Through the Lens of Intersectionality: Sexual Minority Refugees in Canada. Canadian Social Work Review; Ottawa, $28(1), 151-156$.

CAMH. (2014). CAMH: Hidden in Our Midst: New Research Study on Homeless Newcomer Youth (p. 36). Toronto. Retrieved from http://www.camh.ca/en/hospital/about_camh/newsroom/news_releases_media_advisories_and_b ackgrounders/current_year/Pages/Hidden-in-Our-Midst-New-Research-Study-on-HomelessNewcomer-Youth.aspx 
Caxaj, C. S., \& Berman, H. (2010). Belonging Among Newcomer Youths: Intersecting Experiences of Inclusion and Exclusion. Advances in Nursing Science, 33(4), E17-E30. https://doi.org/10.1097/ANS.0b013e3181fb2f0f

Cohen, B. J. (2016). Mental health support for newcomer youth: Fostering resilience and compassion among refugees and their peers (M.A.). University of Toronto (Canada), Canada. Retrieved from http://search.proquest.com.ezproxy.lib.ryerson.ca/docview/1814234818/abstract/503DF6731589 $49 \mathrm{ACPQ} / 1$

Crenshaw, K. (1991). Mapping the Margins: Intersectionality, Identity Politics, and Violence against Women of Color. Stanford Law Review, 43(6), 1241-1299. https://doi.org/10.2307/1229039

Creswell, J. W. (2012). Qualitative Inquiry and Research Design: Choosing Among Five Approaches. SAGE Publications.

Curran, A., Bowness, E., Comack, E., \& Canadian Centre for Policy Alternatives. Manitoba Office. (2010). Meeting the needs of youth: perspectives from youth-serving agencies. Canadian Centre for Policy Alternatives. Manitoba Office.

Daley, A., Solomon, S., Newman, P. A., \& Mishna, F. (2008). Traversing the Margins:

Intersectionalities in the Bullying of Lesbian, Gay, Bisexual and Transgender Youth. Journal of Gay \& Lesbian Social Services, 19(3-4), 9-29. https://doi.org/10.1080/10538720802161474

Deckers, C. M., \& Zinga, D. (2012). Locating Home: Newcomer Youths' School and Community Engagement. Canadian Journal of Education; Toronto, 35(3), 30-47.

Dix, D. B., Klassen, A. F., Papsdorf, M., Klaassen, R. J., Pritchard, S., \& Sung, L. (2009). Factors affecting the delivery of family-centered care in pediatric oncology. Pediatric Blood \& Cancer, 53(6), 1079-1085. https://doi.org/10.1002/pbc.22168 
Francis, J., \& Yan, M.-C. (2016). Bridging the Gaps: Access to Formal Support Services among Young African Immigrants and Refugees in Metro Vancouver. Canadian Ethnic Studies; Calgary, 48(1), 77-100.

Gaytan, F. X., Carhill, A., \& Suarez-Orozco, C. (2007). Understanding and Responding to the Needs of Newcomer Immigrant Youth and Families. Prevention Researcher, 14(4), 10-13.

Ghabrial, M. A. (2017). “Trying to Figure Out Where We Belong”: Narratives of Racialized Sexual Minorities on Community, Identity, Discrimination, and Health. Sexuality Research and Social Policy, 14(1), 42-55. https://doi.org/10.1007/s13178-016-0229-х

Hughes, L. C., \& Preski, S. (1997). Using key informant methods in organizational survey research: Assessing for informant bias. Research in Nursing \& Health, 20(1), 81-92. https://doi.org/10.1002/(SICI)1098-240X(199702)20:1<81::AID-NUR9>3.0.CO;2-Q

Kainer, J. (2016). Intersectionality at Work: Young Women Organizers' Participation in Labour Youth Programs in Canada. Resources for Feminist Research Journal/ Revue de Documentation Sur La Recherche Féministe, 34(3\&4). Retrieved from http://jps.library.utoronto.ca/index.php/rfr-drf/article/download/27574

Khanlou, N., \& Crawford, C. (2006). Post-Migratory Experiences of Newcomer Female Youth: SelfEsteem and Identity Development. Journal of Immigrant and Minority Health, 8(1), 45-56. https://doi.org/10.1007/s10903-006-6341-x

Khanlou, N., Shakya, Y., \& Muntaner, C. (2009). Mental health services for newcomer youth: exploring needs and enhancing access. The Provincial Centre of Excellence for Child and Youth Mental Health.

King, G., Canada, \& Citizenship and Immigration Canada. (2012). Barriers to health service utilization by immigrant families raising a disabled child: unmet needs and the role of 
discrimination. Ottawa, Ont.: Citizen and Immigration Canada. Retrieved from http://www.deslibris.ca/ID/232159

Lee, E. O. J., \& Brotman, S. (2013). Structural Intersectionality and Anti-Oppressive Practice with LGBTQ Refugees in Canada. Canadian Social Work Review; Ottawa, 30(2), 157-183.

Malcolm, Z. T., \& Mendoza, P. (2014). Afro-Caribbean International Students' Ethnic Identity Development: Fluidity, Intersectionality, Agency, and Performativity. Journal of College Student Development, 55(6), 595-614. https://doi.org/10.1353/csd.2014.0053

Mihai, M., \& Burciu, A. (2015). NEET and Youth Exclusion. Romanian Economic Journal, XVIII(56), 135-146.

Moss, A. (2008). Community Arts Programming as a Factor in Newcomer Youth Inclusion: The Toronto Situation. Ryerson University.

Neuman, D. (2014). Qualitative research in educational communications and technology: a brief introduction to principles and procedures. Journal of Computing in Higher Education, 26(1), 6986. https://doi.org/10.1007/s12528-014-9078-x

Núñez, A.-M. (2014). Employing Multilevel Intersectionality in Educational Research: Latino Identities, Contexts, and College Access. Educational Researcher, 43(2), 85-92. https://doi.org/10.3102/0013189X14522320

Núñez, A.-M. (2016). Advancing an Intersectionality Framework in Higher Education: Power and Latino Postsecondary Opportunity. In Higher Education: Handbook of Theory and Research (pp. 33-92). Springer Netherlands.

Omidvar, R., \& Richmond, T. (2003). Immigrant settlement and social inclusion in Canada. Toronto: Laidlaw Foundation. 
Ontario Healthy Communities Coalition. (2004). Inclusive Community Organizations: A Tool Kit.

Retrieved from http://www.ohcc-ccso.ca/en/inclusive-community-organizations-a-tool-kit

Oxman-Martinez, J., \& Choi, Y. R. (2014). Newcomer Children: Experiences of Inclusion and Exclusion, and Their Outcomes. Social Inclusion, 2(4), 23-37.

Patel, P., \& Siddiqui, H. (2007). Standing in the Same Dream: Black and Minority Women's Struggles Against Gender-Based Violence and for Equality in the UK. In Gender Orders Unbound: Globalisation, Restructuring and Reciprocity (pp. 259-276). Barbara Budrich. Refugee Support \& Metropolitan Trust. (2009). Over Not Out: The housing and homelessness issues specific to lesbian, gay, bisexual and transgender asylum seekers. (p. 28). Retrieved from https://thefemilist.wordpress.com/2009/05/22/resource-over-not-out-the-housing-andhomelessness-issues-specific-to-lesbian-gay-bisexual-and-transgender-asylum-seekers/

Strandbu, Å. (2005). Identity, embodied culture and physical exercise: Stories from Muslim girls in Oslo with immigrant backgrounds. YOUNG, 13(1), 27-45. https://doi.org/10.1177/1103308805048751

Suárez-Orozco, C., Yoshikawa, H., \& Tseng, V. (2015). Intersecting Inequalities: Research to Reduce Inequality for Immigrant-Origin Children and Youth. William T. Retrieved from https://eric.ed.gov/?id=ED568399

Thomas, C. (2012). Newcomers and Social Inclusion in Peel Region, Ontario: Examining the Importance of Settlement Services (Thesis). Retrieved from https://tspace.library.utoronto.ca/handle/1807/33563

Travers, R., Guta, A., Flicker, S., Larkin, J., Lo, C., McCardell, S., \& van der Meulen, E. (2010). Service provider views on issues and needs for lesbian, gay, bisexual, and transgender youth. The Canadian Journal of Human Sexuality; Toronto, 19(4), 191-198. 
Van der Meulen, E., Oliver, V., Flicker, S., \& Travers, R. (2010). Obstacles to sexual health services for youth: Service providers' perspectives. The Canadian Journal of Human Sexuality; Toronto, 19(4), 185-190.

Van Ngo, H. (2009). Patchwork, Sidelining and Marginalization: Services for Immigrant Youth. Journal of Immigrant \& Refugee Studies, 7(1), 82-100.

https://doi.org/10.1080/15562940802687280

Warner, L. R., \& Shields, S. A. (2013). The Intersections of Sexuality, Gender, and Race: Identity Research at the Crossroads. Sex Roles, 68(11-12), 803-810. https://doi.org/10.1007/s11199-0130281-4

Yee, J. Y., Marshall, Z., \& Vo, T. (2014). Challenging Neo-Colonialism and Essentialism: Incorporating Hybridity into New Conceptualizations of Settlement Service Delivery with Lesbian, Gay, Bisexual, Trans, and Queer Immigrant Young People. Critical Social Work, 15(1). Retrieved from http://www1.uwindsor.ca/criticalsocialwork/challenging_neocolonialism

Yi, J., Kim, M. A., Kim, K., \& Hong, J. S. (2016). Exploring Social Service Providers' Perspectives on Barriers to Social Services for Early Adjustment of Immigrant Adolescents in South Korea. Journal of Immigrant and Minority Health, 18(5), 1076-1084. https://doi.org/10.1007/s10903016-0406-2 